\title{
Econometrics of Ascending Auctions by Quantile Regression
}

\section{NATHALIE GIMENES}

WorkING PAPER SERIES № 2014-25 


\title{
DEPARTMENT OF ECONOMICS, FEA-USP
}

WORKING PAPER № 2014-25

\section{Econometrics of Ascending Auctions by Quantile Regression}

\author{
Nathalie Gimenes (ncgmsanches@gmail.com)
}

\begin{abstract}
:
This paper suggests an identification and estimation approach based on quantile regression to recover the underlying distribution of bidders' private values in ascending auctions under the IPV paradigm. The quantile regression approach provides a flexible and convenient parametrization of the private values distribution, with an estimation methodology easy to implement and with several specification tests. The quantile framework provides a new focus on the quantile level of the private values distribution and on the seller's optimal screening level, which can be both useful for bidders and seller. The empirical application on timber auctions suggests that using policy recommendations from seller's expected payoff may be sometimes inappropriate from a seller's point of view due to the low probability of selling the good. This seems to be an important issue specially in auctions with strong heterogeneity among the bidders, since the seller has incentive to screen bidders' participation by setting a high reservation price, which in turn leads to a low probability of selling the good.
\end{abstract}

Keywords: Private values; timber auctions; ascending auctions; seller expected revenue; quantile regression identification; quantile regression estimation; quantile regression specification testing.

JEL Codes: C14; D44; L70 


\title{
Econometrics of Ascending Auctions by Quantile Regression
}

\author{
Nathalie Gimenes \\ Department of Economics, University of São Paulo, Brazil.
}

October 2014

\begin{abstract}
This paper suggests an identification and estimation approach based on quantile regression to recover the underlying distribution of bidders' private values in ascending auctions under the IPV paradigm. The quantile regression approach provides a flexible and convenient parametrization of the private values distribution, with an estimation methodology easy to implement and with several specification tests. The quantile framework provides a new focus on the quantile level of the private values distribution and on the seller's optimal screening level, which can be both useful for bidders and seller. The empirical application on timber auctions suggests that using policy recommendations from seller's expected payoff may be sometimes inappropriate from a seller's point of view due to the low probability of selling the good. This seems to be an important issue specially in auctions with strong heterogeneity among the bidders, since the seller has incentive to screen bidders' participation by setting a high reservation price, which in turn leads to a low probability of selling the good.
\end{abstract}

JEL-Codes: C14, D44, L70

Keywords: Private values; timber auctions; ascending auctions; seller expected revenue; quantile regression identification; quantile regression estimation; quantile regression specification testing.

I am grateful to Emmanuel Guerre for his insightful comments and suggestions. I acknowledge Leonardo Rezende, João Santos Silva, Quang Vuong, Isabelle Perrigne, Valentina Corradi, Martin Pesendorfer, Rafael Coutinho Lima, Frank Verboven and Geert Dhaene for helpful discussions. I would like to thank the School of Economics and Finance, Queen Mary University of London, for generous funding. All remaining errors are my responsibility. 


\section{Introduction}

In an auction theoretical game, the bidders' private value cumulative distribution function (c.d.f.) is a key element for analysing the demand that a seller faces. Because it is required in the computation of the seller's expected payoff, its knowledge is crucial for policy recommendation, as e.g. the optimal reservation price policy. The issue here is that bidders' private values are not observed, thereby their distribution function is unknown for the econometricians and policy makers. In the past 20 years, several structural researches in auction theory have proposed parametric, semiparametric and nonparametric approaches to estimate such a latent distribution function.

The goal of this paper is to propose an identification and estimation approach based on quantile regression to recover the private value conditional distribution in ascending auctions. The identification strategy is developed for the Independent Private Values (IPV) setup, but can also be extended for other paradigms. The ascending auction is one of the most common design in practice and is especially suitable for the identification of the private value distribution under the IPV paradigm because, under mild assumptions, the transaction price equals the second-highest private value. As a result, the private value distribution can be nonparametrically identified through the winning bids distribution, as well known from Athey and Haile (2002).

A first wave of researchers has focused on parametric identification and estimation approaches. Paarsch (1992) and Donald and Paarsch (1993, 1996) have proposed to estimate the parameters of the private value distribution via the method of Maximum Likelihood (ML) using the winning bids of a button auction. In an attempt to circumvent the difficulties that arise from ML methods, Laffont, Ossard and Vuong (1995) suggested simulated method of moments based on the revenue equivalence theorem, which can handle a larger

class of auction mechanisms and parametric models. A key observation of Rezende (2008) is 
that Laffont, Ossard and Vuong (1995)'s approach carries over with drastic simplifications for simple semiparametric linear regression models which can still be identified through the revenue equivalence theorem. More recently, taking into consideration endogenous entry and unobserved heterogeneity, Li and Zheng (2009) propose a semiparametric Bayesian method to estimate the distributions of costs, entry costs and of the unobserved auction heterogeneity. Athey, Levin and Seira (2011) also consider an entry stage and adopt a parametric approach to estimate the distribution of the unobserved heterogeneity. Roberts and Sweeting (2012) investigate parametrically the relative revenue performance of a simultaneous outcry auction and a sequential game when entry is costly and selective.

Some works have proposed nonparametric identification and estimation approaches for ascending auctions in an attempt to circumvent the misspecification bias of parametric ones. Athey and Haile (2002) have shown that the c.d.f of the transaction price can nonparametrically identify the private value distribution. Haile and Tamer (2003) identify bounds on the private value distribution for a model of English auction in which bids are below bidder's value. They also suggest to use a median regression in the estimation as an alternative to the nonparametric estimation method, but not really developed the idea.

Some other nonparametric approaches for first-price sealed-bid auctions have been built on quantiles, starting from the insight of Haile, Hong and Shum (2003) that the quantiles of the private value distribution can be written as a function of the quantiles of the observed bids distribution and density function. Marmer and Shneyerov (2012) have shown that adopting a quantile approach makes the estimation of the private value p.d.f. easier from a technical point of view, whereas Guerre and Sabbah (2012) go one step further by proposing to estimate quantile function instead of p.d.f.. Marmer, Shneyerov and Xu (2013) proposed a nonparametric approach to test for alternative models of entry by exploring variation in the quantiles of the private value distribution due to competition. Enache and Florens (2012) developed a nonparametric approach for third-price auctions under risk aversion. 
To my knowledge, quantile approaches have not been systematically and throughly applied to ascending auctions so far. Although nonparametric approaches have the advantages of being flexible in analysing the data at hand since no structure is imposed on that, it has some disadvantages as the curse of dimensionality and the need to choose for a bandwidth parameter. The curse of dimensionality can be indeed a relevant estimation issue in view of important contributions to the empirical auction literature such as Haile and Tamer (2003) and Aradillas-Lopez, Gandhi and Quint (2013), which consider, respectively, 5 and 6 explanatory variables for a sample size of a few thousands at best. Hastie and Tibshirani (1990) give a convenient example of the curse of dimensionality. Suppose that 1000 points are uniformly distributed over a 5-dimensional unit cube and we wish to construct a cubeshaped neighbourhood containing 10 observations, that is, $1 \%$ of the data. The subcube is then required to have length 0.40 , on average. If instead of 5 the covariate dimension was set to 1 , the length required in a similar exercise would now be $1 \%$ of the covariate range, which is much smaller and then local than the $40 \%$ of the 5 dimensional case. To improve accuracy of the estimates, is then required to use kernels with very small bandwidths and far more observations. This suggests that the bias in standard nonparametric approaches can be high considering the usual auction sample sizes. By contrast, the quantile regression model used in this paper can be in principle estimated with a parametric rate, independently of the dimension of the covariate, and does not involve the choice of a smoothing parameter.

Addressing the curse of dimensionality is an important step to develop a nonparametric framework allowing for many covariates. This is also a first step to better capture unobserved heterogeneity by increasing the number of covariates in a nonparametric framework. Specific quantile techniques have also been developed to deal with omitted variables using instrumental variables, see Chernozhukov and Hansen (2006) among others. Such approaches have natural sieve extensions which could also fit in extensions of the framework studied here. Although not so clearly related with the structure of auction data, quantile panel data 
estimation techniques has provided important recent development, see e.g. Canay (2011) and Galvao Jr. (2011). This suggests that nonparametric quantile approach could be much more useful regarding unobserved heterogeneity than other nonparametric methods.

The quantile regression approach is also more flexible than many of the parametric or semiparametric methods mentioned above. Indeed, the model includes functional components that may be helpful to reduce the impact of misspecification. Compared to the semiparametric regression approach of Rezende (2008), quantile regression is computationally more difficult to perform but delivers an estimation of the full private value distribution, as needed for instance to derive an optimal reservation price. As a consequence, quantile regression is probably better suited for policy recommendations than a simpler regression approach. Also in the context of policy analysis, the quantile regression approach allows to highlight the screening level implied by the choice of a reservation price, a policy characteristic that has been mostly ignored by previous empirical approaches. Based on data from the United States Forest Service (USFS) timber auctions, the empirical application suggests that practical implementation of the optimal reservation price derived from maximization of the expected payoff may lead to a low probability of selling the auctioned good. That is due to a strong variation observed in the private value conditional quantiles, which may then incentive sellers to screen bidders with low valuation in the auction.

The paper is organized as follows. Section 2 describes the ascending auction considered, the quantile regression identification approach and a quantile version for the seller's expected payoff with a corresponding screening level maximizer. Section 3 provides the estimation methodology and studies the performance of the estimator in small and large-samples, allowing for potential misspecification. Section 4 presents some specification tests. Section 5 provides an empirical application of the methodology proposed. Finally, section 6 concludes the paper. Appendix A groups all the proofs of the results achieved, Appendix B describes the random weighting bootstrap method considered in two of the hypotheses tests 
and Appendix C presents Monte Carlo experiments of the hypotheses tests.

\section{Ascending Auctions in a Quantile Setup}

A single and indivisible object with some characteristics $Z \in \mathbb{R}^{d}$ is auctioned to $I \geq 2$ bidders through an ascending auction. The seller sets a reservation price $r$ prior to the auction that is the minimum price that he would be willing to accept. Both the set of auction covariates $X=(1, Z)$ and the number of actual bidders $I$ participating in the auction are common knowledge. The object is sold to the highest bidder for the price of his last bid, provided that it is at least as high as the reservation price $r$. Within the IPV paradigm, each bidder $i=1, \cdots, I$ is assumed to have a private value $v_{i}$ for the auctioned good, which is not observed by other bidders. The bidder only knows his own private value, but it is common knowledge for bidders and sellers that private values have been identically and independently drawn from a common c.d.f. $F_{v}(\cdot \mid X, I)$ conditional upon $(X, I)$, or equivalently, with a conditional quantile function $V(\alpha \mid X, I), \alpha \in[0,1]$, defined as

$$
V(\alpha \mid X, I):=\inf \left\{v: F_{v}(v \mid X, I) \geq \alpha\right\}
$$

When the private value conditional distribution is absolutely continuous with a probability density function (p.d.f) $f_{v}(\cdot \mid X, I)$ positive on its support $[V(0 \mid X, I), V(1 \mid X, I)] \subset \mathbb{R}+$, as considered from now on, $V(\alpha \mid X, I)$ is the reciprocal function $F_{v}^{-1}(\alpha \mid X, I)$.

It is well known ${ }^{1}$ that $u_{i}=F_{v}\left(v_{i} \mid X, I\right)$, which can be viewed as the rank of a bidder with private value $v_{i}$ in the population, is independent of $(X, I)$ with a uniform distribution over $[0,1]$. The IPV paradigm implies that the ranks $u_{i}, i=1, \cdots, I$, are independent. In other words, the dependence between the private values $v_{i}$ and the auction covariates $(X, I)$ can

\footnotetext{
${ }^{1}$ By the Fundamental Theorem of Simulation.
} 
be fully captured by the nonseparable model

$$
v_{i}=V\left(u_{i} \mid X, I\right), \quad u_{i} \stackrel{i i d}{\sim} \mathcal{U}_{[0,1]} \perp(X, I)
$$

Therefore, bidders are identical up to the variable $u_{i}$, which represents the bidder $i$ th's position in the private value distribution.

The quantile regression approach, developed by Koenker and Bassett (1978), restricts the quantile representation (2.2) to a regression specification, such as

$$
\begin{aligned}
V(\alpha \mid X, I) & =h(X \gamma(\alpha \mid I)) \\
& =h\left(\gamma_{0}(\alpha \mid I)+Z \gamma_{1}(\alpha \mid I)\right)
\end{aligned}
$$

where $h(\cdot)$ is a given function, $\gamma_{0}(\alpha \mid I)$ the quantile regression intercept and $\gamma_{1}(\alpha \mid I)$ the quantile regression slopes. In the basic specification, $h(\cdot)$ is equal to the identity. Note that in (2.3), both the intercept and the slope quantile regression coefficients depend upon the rank $\alpha$ of the bidder in the population ${ }^{2}$. Therefore, changes in the conditioning variables not only shift the location of the conditional distribution of $v$, but may also affect its scale and shape. A shock on the covariate $X$ may affect a bidder with a low rank $\alpha$ in a different way than a bidder with a higher rank. A large discrepancy of the coefficients across $\alpha$ indicates strong heterogeneity ${ }^{3}$ among the bidders. As discussed later, taking into consideration such heterogeneity among the bidders may have important implications for both seller and bidders.

I now turn to the assumptions of the model. In the considered ascending auction, bidders raise continuously their prices and drop out of the auction as the prices reach their valuation.

Assumption 1 The transaction price in an auction is the greater of the reservation price

\footnotetext{
${ }^{2}$ Throughout this paper, the rank of the bidder can be interchangeably represented by $\alpha$ and $u$.

${ }^{3}$ Note that heterogeneity and asymmetry are two concepts that should not be confused. Heterogeneity is concerned with the variation of $\gamma(\alpha)$ across quantile levels $\alpha$, while asymmetry implies that different bidders can have different coefficients $\gamma(\alpha)$.
} 
and the second-highest bidder's willingness to pay.

Assumption 1 is an assumption on equilibrium play. This assumption was also used in Aradillas-Lopez et al. (2013) and, as noted in Athey and Haile (2002) and Bikhchandani, Haile and Riley (2002), is compatible with the multiple equilibria generated by the ascending auctions. It is for instance the result of the dominant strategy equilibrium of a button ${ }^{4}$ auction, which is a stylized version of an ascending auction. Haile and Tamer (2003) use stronger assumptions concerning bidder's behaviour, which determine the joint distribution of all the bids. This is not needed when using only the winning bid. This assumption would also hold approximately in the context of Haile and Tamer (2003) if bidders do not use jump bids at the end of the auction.

The ascending auction format is specially convenient for the identification of the bidders' private value distribution under the IPV paradigm ${ }^{5}$ because, under assumption 1 and a nonbinding reservation price, the latent private value conditional distribution can be nonparametrically identified from the winning bid conditional distribution. Such a nice feature will be considered in the identification of the model.

The next two assumptions deal with the quantiles of the bidders' private value distribution:

Assumption $2 V(\alpha \mid X, I)$ is strictly increasing and continuous on its support $[V(0 \mid X, I), V(1 \mid X, I)]$ for $\operatorname{all}(X, I)$.

Assumption 3 The private value conditional quantiles has a quantile regression specification

$$
V(\alpha \mid X, I)=h(X \gamma(\alpha \mid I)),
$$

\footnotetext{
${ }^{4}$ In a button auction, bidders hold down a button to remain active while the price rises continuously, releasing the button to drop out of the game, and the willingness to pay of the losing bidders is learned from their drop out prices.

${ }^{5}$ In the case of affiliated private values, the private value conditional quantiles cannot be nonparametrically point-identified. It is possible however to extend the methodology to identify bounds on the private value conditional quantiles using a strategy similar to Aradillas-Lopez et al. (2013).
} 
where $h(\cdot)$ is known by the econometrician.

Assumption 2 is usual in the quantile regression literature. Assumption 3 imposes correct specification of the private value conditional quantiles as necessary for our identification results. This will be relaxed when studying estimation of the model.

As shown in the next Lemma, the rank $\alpha$ of a bidder in the population has a direct relationship with his probability of winning the auction, so that estimating $V(\alpha \mid X, I)$ can be helpful for newcomers that do not know the market a priori and used as a benchmark to achieve a desired probability of winning the auction.

Lemma 1 Under the IPV paradigm and assumptions 1-2, a bidder with private value $V(\alpha \mid X, I)$ wins with probability $\alpha^{I-1}$.

The proof of Lemma 1 is given in Appendix A, which also groups the proof of all the results stated in this paper.

Define

$$
\Psi_{I}(t)=I t^{I-1}-(I-1) t^{I}
$$

and let $B(\alpha \mid X, I)$ be the $\alpha$-quantile of the winning bids conditional distribution given $(X, I)$. It follows from Athey and Haile (2002, equation (5)) that $\Psi_{I}\left(F_{v}(\cdot \mid X, I)\right)$ is the distribution of the second-highest private value, which is equal to the winning bid. This gives the following cornerstone quantile identification result.

Lemma 2 Under IPV and assumptions 1-2, for each $I$ and $\alpha \in[0,1]$,

$$
V(\alpha \mid X, I)=B\left(\Psi_{I}(\alpha) \mid X, I\right)
$$

Lemma 2 shows that the private value conditional quantile function can be nonparametrically identified by the conditional quantile of the observed winning bid. In the next section, a 
method to estimate $V(\cdot \mid X, I)$ will be proposed. Before introducing the quantile regression identification result, the following assumption for the auction specific covariate $X=(1, Z)$ is required:

Assumption 4 The auction specific variable, Z, has dimension d, with a compact support in $\mathcal{Z} \subset(0,+\infty)^{d}$ and a nonempty interior.

Assumption 4 ensures that if $x \gamma_{1}=x \gamma_{2}$, for all $x \in \mathcal{X}$, thus $\gamma_{1}=\gamma_{2}$, and is necessary for the quantile regression identification below.

Lemma 3 Under IPV and assumptions 1-4,

(i) There exists, for each $\alpha \in[0,1]$, a vector of coefficients $\beta(\alpha \mid I)$ such that

$$
B(\alpha \mid X, I)=h(X \beta(\alpha \mid I))
$$

(ii) $\beta(\alpha \mid I)$ is uniquely defined and satisfies

$$
\beta\left(\Psi_{I}(\alpha) \mid I\right)=\gamma(\alpha \mid I)
$$

Result (i) in Lemma 3 is a stability property of the quantile regression specification, which is a consequence of Lemma 2. Indeed, Lemma 2 shows that the winning bid quantile function admits the same specification that the one postulated for the private values, but for a transformed quantile level. Lemma 3-(ii) gives the identification result of the quantile regression approach. It shows that the coefficient $\gamma(\cdot \mid I)$ of the private value conditional quantile function is identified through the coefficient $\beta(\cdot \mid I)$ of the winning bid conditional quantile function, but evaluated at a different quantile level $\Psi_{I}(\cdot)$. 


\subsection{Dependence upon Bidders Participation}

It is assumed for now that the private value distribution is conditional upon the number of bidders $I$ participating in the auction. Although standard in many econometric works as Guerre, Perrigne and Vuong (2000) among others, conditioning on $I$ is not usual in theoretical auction models, see e.g. Krishna (2010). This choice can be however motivated by the three following reasons which consider either unobserved heterogeneity or an entry stage.

A first setting of interest is unobserved heterogeneity. Instead of the observed $X$, the bidders use an auction characteristic $\left(X, X_{u}\right)$ which includes a component $X_{u}$ that is not observed by the econometrician. Hence, the private value quantile relevant for policy analysis is $V\left(\alpha \mid X, X_{u}\right)$, which cannot be estimated without further assumption. It can be for instance assumed that the actual number of bidders depends upon the auction characteristic, that is $I=I\left(X, X_{u}\right)$, in a way that fully captures the impact of the unobserved characteristic, i.e. $V\left(\cdot \mid X, X_{u}\right)=V\left(\cdot \mid X, I\left(X, X_{u}\right)\right)$ so that the conditional quantile $V(\cdot \mid X, I)$ is fully relevant for policy analysis purposes.

A second motivation is given by the recent econometric literature on entry, see Gentry and Li (2012), Li and Zheng (2009) and Marmer et al. (2013). These models consider a two stage game, where the first stage is entry and the second stage is the auction game. The structural parameter is the joint distribution of the private values and signals given the characteristic $X$, which is used in the entry stage of the game. The second stage involves an actual number of bidders $I$, who have decided to participate in the auction, and the conditional quantile $V(\cdot \mid X, I)$ of private values given $X$ and $I$. A key contribution of the aforementioned econometric literature is that the structural parameter is identified from $I$ and $V(\cdot \mid X, I)$, so that estimation of the model can be performed through estimation of the conditional c.d.f. or quantile of private values given $X$ and $I$.

A third motivation notes that, whereas the reality of the auction is physically clear, the 
entry stage of the game described above may have a more conceptual nature. Therefore, in some cases, the importance of a entry stage is an assumption that should be tested by investigating whether $V(\cdot \mid X, I)$ depends indeed upon $I$. A test built on the null hypothesis of independence, i.e. $V(\cdot \mid X)=V(\cdot \mid X, I)$, is proposed in section 4.1 and then applied in the empirical section.

\subsection{Optimal Reservation Price}

Consider a binding reservation price set by the seller, i.e. $r(X, I) \in[V(0 \mid X, I), V(1 \mid X, I)]$. The reservation price thus plays the role of a screening level in the auction since bidders with $V\left(\alpha_{i} \mid X, I\right)<r(X, I)$ are prevented from participating in the game. Let $\alpha_{r}$ be the screening level in the private value conditional distribution, i.e. $\alpha_{r}$ is such that $r(X, I)=V\left(\alpha_{r} \mid X, I\right)$. It thus represents the percentage of bidders in the population that are not participating in the auction because of a low valuation. Note that the auctioned good will not be sold if all the players have valuation below $r(X, I)$, which implies that the probability of trading is $1-\alpha_{r}^{I}$. Therefore, for a given $I$, the probability of trading decreases with the screening level $\alpha_{r}$.

Let the seller's payoff be defined as

$$
\pi(r(X, I))=b_{w} \mathbb{I}\left(b_{w} \geq r(X, I)\right)+v_{0}(X)\left(1-\mathbb{I}\left(b_{w} \geq r(X, I)\right)\right)
$$

where $b_{w}$ is the winning bid and $v_{0}(X)$ the seller's private value ${ }^{6}$. In what follows, let $v_{0}=v_{0}(X)$ and $r=r(X, I)$. The following proposition gives a quantile version for the seller's expected payoff, a candidate for the optimal screening level $\alpha_{r}^{*}=\alpha_{r}^{*}\left(X, I, v_{0}\right)$ and the corresponding optimal reservation price $V\left(\alpha_{r}^{*} \mid X, I\right)$. Let $\Pi\left(\alpha_{r} \mid X, I, v_{0}\right)$ be the seller's expected payoff given $(X, I)$ when the screening level is $\alpha_{r}$.

\footnotetext{
${ }^{6}$ The seller's profit is given by his payoff minus his private value. I focus the analysis on the seller's payoff.
} 
Proposition 4 Under IPV and assumptions 1-2,

(i) the seller's expected payoff is

$$
\begin{aligned}
\Pi\left(\alpha_{r} \mid X, I, v_{0}\right)=v_{0} \alpha_{r}^{I} & +V\left(\alpha_{r} \mid X, I\right) I \alpha_{r}^{I-1}\left(1-\alpha_{r}\right) \\
& +I(I-1) \int_{\alpha_{r}}^{1} V(\alpha \mid X, I) \alpha^{I-2}(1-\alpha) d \alpha,
\end{aligned}
$$

where $v_{0}$ is the seller's private value;

(ii) The optimal reservation price $V\left(\alpha_{r}^{*} \mid X, I\right)$ satisfies

$$
V\left(\alpha_{r}^{*} \mid X, I\right)-V^{(1)}\left(\alpha_{r}^{*} \mid X, I\right)\left(1-\alpha_{r}^{*}\right)=v_{0},
$$

where $V^{(1)}\left(\alpha_{r}^{*} \mid X, I\right)$ is the private value quantile density function.

Equation (2.8) in Proposition 4 gives the seller's expected payoff in a quantile setup. It differs from the expression given in Riley and Samuelson (1981, Proposition 1) because it does not involve the private value conditional density. It is nevertheless convenient for estimation purposes because, in a nonparametric setup, $V(\cdot \mid X, I)$ can be estimated in a faster rate than $V^{(1)}(\cdot \mid X, I)$. Equation (2.9) is the first-order condition (FOC) associated with the maximization of the seller's expected payoff and represents a quantile version of a well known formula that states that the optimal reservation price satisfies $1-F_{v}\left(r^{*} \mid X, I\right)-$ $\left(r^{*}-v_{0}\right) f_{v}\left(r^{*} \mid X, I\right)=0$, see Krishna (2010, p.23), Riley and Samuelson (1981, Proposition 3) and Myerson (1981). An interesting implication of (2.9) is that when $V(\alpha \mid X, I)$ does not depend upon $I$ the optimal reservation price $V\left(\alpha_{r}^{*} \mid X\right)$ and the optimal screening level $\alpha_{r}^{*}$ also do not depend on $I$, as well known from the aforementioned reference. In addition,

\footnotetext{
${ }^{7}$ The quantile density function is defined as the derivative of the quantile function with respect to $\alpha$, i.e. $V^{(1)}(\alpha \mid X, I)=\partial V(\alpha \mid X, I) / \partial \alpha=1 / f_{v}(V(\alpha \mid X, I) \mid X, I)$.
} 
since $V^{(1)}(\cdot \mid X, I)>0$, it is clear that $\alpha_{r}^{*}>\alpha_{0}$, where $v_{0}=V\left(\alpha_{0} \mid X, I\right)$. That is, the optimal reservation price is above $v_{0}$.

Although equation (2.9) in Proposition 4 gives a closed form to estimate the optimal reservation price $V\left(\alpha_{r}^{*} \mid X, I\right)$, it involves an estimation of the quantile density function $V^{(1)}(\cdot \mid X, I)$. It is, therefore, better to maximize an estimation of $\Pi\left(\alpha_{r} \mid X, I, v_{0}\right)$ to get $\widehat{\alpha}_{r}^{*}$ than to solve an estimation of the quantile FOC ${ }^{8}$. For the estimation of the seller's expected payoff, I first estimate $V(\cdot \mid X, I)$ using the quantile regression methodology proposed in the next section and then apply numerical integration via a trapezoildal rule over a grid of quantiles $\mathcal{A}=\left\{\alpha_{1}, \alpha_{2} \cdots, \alpha_{K}\right\}^{9}$ to estimate the definite integral in (2.8). The optimal screening level $\widehat{\alpha}_{r}^{*}$ is then chosen over $\mathcal{A}$ such that $\widehat{\Pi}\left(\alpha_{r} \mid X, I, v_{0}\right)$ is maximized, where the latter is an estimation of $\Pi\left(\alpha_{r} \mid X, I, v_{0}\right)$. As mentioned above, when $V(\cdot \mid X)=V(\cdot \mid X, I)$, the optimal screening level does not depend upon $I$. In the empirical section, this case is considered and $\widehat{\alpha}_{r}^{*}$ is therefore estimated by maximizing an aggregate $\widehat{\Pi}\left(\alpha_{r} \mid X, v_{0}\right)$, i.e.

$$
\widehat{\alpha}_{r}^{*}=\arg \max \sum_{I=2}^{\bar{I}} \widehat{\Pi}\left(\alpha_{r} \mid X, I, v_{0}\right) L_{I} / L
$$

where $\{2, \cdots, \bar{I}\}$ is the support of $I, L_{I}$ is the number of auctions in the sample with $I$ bidders competing and $L$ the total sample size. The intuition here is that aggregating over $I$ may potentially give a better estimation $\widehat{\alpha}_{r}^{*}$.

\footnotetext{
${ }^{8} \mathrm{Li}$, Perrigne and Vuong (2003) have also used the same strategy to estimate the optimal reservation price in the case of affiliated private values, which includes the IPV as a special case. They define such a strategy as semiparametric, since the optimal reservation price is obtained as the maximizer of an estimated expected payoff in which the distributions and densities were nonparametrically estimated in a first step.

${ }^{9}$ In the empirical application, the grid of prescribed quantiles used for this numerical integration is $\mathcal{A}=\{0.12,0.14, \cdots, 0.80\}$.
} 


\section{Estimation Methodology}

Consider independent and identically distributed observations $\left(b_{w_{\ell}}, Z_{\ell}, \ell=1, \ldots, L\right)$, where $b_{w_{\ell}}$ is the winning bid at auction $\ell$, and $Z_{\ell}$ a specific characteristic of the good auctioned in auction $\ell$. Let $X_{\ell}=\left(1, Z_{\ell}\right) \in \mathcal{X}$ be a row vector of dimension $d+1$ and $\mathcal{X}=\{1\} \times \mathcal{Z}$. Define $L_{I}$ as the number of auctions with $I$ players,

$$
L_{I}=\sum_{\ell=1}^{L} \mathbb{I}\left(I_{\ell}=I\right)
$$

for $I \in \mathcal{I}$, where $\mathcal{I}$ is a bounded subset of $\{2,3, \ldots\}$.

The following assumption concerns the variables in our model:

Assumption 5 The variables $\left\{I_{\ell}, X_{\ell}, v_{i \ell}, i=1,2, \ldots I_{\ell}, \ell=1, \ldots, L\right\}$ are independent and identically distributed. Conditional on $\left(X_{\ell}, I_{\ell}\right)$, the private values $v_{i \ell}$ are independent with common c.d.f. $F_{v}\left(\cdot \mid X_{\ell}, I_{\ell}\right)$ and a density function $f_{v}\left(\cdot \mid X_{\ell}, I_{\ell}\right)$ bounded away from zero.

Assumption 5 implies that each auction is independent and that, within an auction, the IPV paradigm holds. Note that it is not assumed that $I_{\ell}$ is independent upon $X_{\ell}$. Recall that $V\left(\cdot \mid X_{\ell}, I_{\ell}\right)=F_{v}^{-1}\left(\cdot \mid X_{\ell}, I_{\ell}\right)$.

Assumption 3 from the preceding section was considering a correct specification of the private value quantile regression model as necessary for identification. This is not required in our estimation setup and Assumption 6 below, which does not assume a correct specification and considers instead the function $h(\cdot)$, will be used ${ }^{10}$ instead of Assumption 3.

Assumption 6 The function $h(t)$ used in the private values regression model is a given continuous, monotonically increasing and twice differentiable function.

\footnotetext{
${ }^{10}$ In the empirical section, two functions in special will be studied: the identity function $h(t)=t$ and the exponential function $h(t)=\exp (t)$.
} 
Consider the winning bid $b_{w_{\ell}}$ at the auction $\ell, \ell=1, \cdots, L$, which is the amount paid by the winner. From the quantile regression identification result (2.6) in Lemma $3, \gamma(\alpha \mid I)$ can be estimated through

$$
\widehat{\gamma}(\alpha \mid I)=\widehat{\beta}\left(\Psi_{I}(\alpha) \mid I\right)
$$

I propose to estimate the winning bid quantile regression population parameter $\beta(\alpha \mid I)$ via the method of quantile regression,

$$
\widehat{\beta}(\alpha \mid I)=\arg \min _{\beta \in \Gamma} \frac{1}{L_{I}} \sum_{\ell=1}^{L_{I}} \mathbb{I}\left(I_{\ell}=I\right) \rho_{\alpha}\left(b_{w_{\ell}}-h\left(X_{\ell} \beta\right)\right)
$$

where $\rho_{\alpha}(u)=u(\alpha-\mathbb{I}(u<0))$ and $\Gamma$ is a compact subset of $\mathbb{R}^{d+1}$. In other words,

$$
\widehat{\gamma}(\alpha \mid I)=\arg \min _{\gamma \in \Gamma} \frac{1}{L_{I}} \sum_{\ell=1}^{L_{I}} \mathbb{I}\left(I_{\ell}=I\right) \rho_{\Psi_{I}(\alpha)}\left(b_{w_{\ell}}-h\left(X_{\ell} \gamma\right)\right)
$$

The next two sections study the performance of the estimator (3.11) in small and large samples, respectively. First, the performance of (3.11) in finite samples is illustrated in comparison with a nonparametric approach via Monte Carlo experiments and then its asymptotic distribution is studied.

\subsection{Finite-Sample Performance}

In this section, the performance in small samples of the quantile regression estimator (3.11) will be studied in comparison with the alternative and most used nonparametric approach via a kernel function. The latter is widely used in the empirical auction literature due to its flexibility in representing the data since no structure is imposed in the analysis. Comparing a nonparametric revenue analysis with a quantile regression one is therefore a way to assess whether the quantile regression is correct from a policy recommendation point of view. 
To achieve this goal, I carry out 5 different types of experiments. In each experiment, a population private value quantile function $V(\alpha \mid X, I)$ is generated and i.i.d. samples of size $I$ are drawn from $V\left(\alpha \mid X_{\ell}, I_{\ell}\right)$ for each auction $\ell, \ell=1, \cdots, L$. The winning bid is given by the second-highest private value. The winning bids are used to estimate the seller's expected revenue and the correspoding optimal reservation price using both estimation approaches. For the quantile regression approach, I estimate the seller's expected revenue for a grid of quantile levels $\mathcal{A}=\left\{\alpha_{1}, \alpha_{2} \cdots, \alpha_{K}\right\}$ an choose $\widehat{\alpha}_{r}$ to maximize (2.8), as suggested in Proposition 4. The optimal reservation price is given by $\widehat{V}\left(\widehat{\alpha}_{r} \mid X, I\right)$.

For the nonparametric approach, the conditional distribution of private values is used instead of their quantiles. Write $\mathbb{P}\left(v_{I: I}<r \mid X, I\right)=F_{v}^{I}(r \mid X, I)$,

$$
\mathbb{P}\left(v_{I-1: I}<r \leq v_{I: I} \mid X, I\right)=F_{b_{w}}(r \mid X, I)-F_{v}^{I}(r \mid X, I)
$$

and

$$
\mathbb{E}\left[v_{I-1: I} \mathbb{I}\left(r \leq v_{I-1: I}\right) \mid X, I\right]=\int_{r}^{\bar{v}} v f_{b_{w}}(v \mid X, I) d v,
$$

where $F_{b_{w}}(\cdot \mid X, I)$ and $f_{b_{w}}(\cdot \mid X, I)$ are, respectively, the c.d.f. and p.d.f. of the winning bids conditional on $(X, I)$. From the proof of Proposition 4, the seller's expected payoff can be written as

$$
\begin{aligned}
\Pi\left(r \mid X, I, v_{0}\right)=v_{0} F_{v}^{I}(r \mid X, I) & +r\left(F_{b_{w}}(r \mid X, I)-F_{v}^{I}(r \mid X, I)\right) \\
& +\int_{r}^{\bar{v}} v f_{b_{w}}(v \mid X, I) d v .
\end{aligned}
$$

To estimate $\Pi\left(r \mid X, I, v_{0}\right)$, I first estimate nonparametrically the winning bid conditional c.d.f. and p.d.f., then apply the transformation $\Psi_{I}^{-1}(\cdot)$ to find the solution for $\widehat{F}_{v}(\cdot \mid Z, I)=$ $\Psi_{I}^{-1}\left(\widehat{F}_{b_{w}}(\cdot \mid Z, I)\right)$ and estimate the integral term via numerical integration as above. The 
nonparametric estimation of $F_{b_{w}}(\cdot \mid X, I)$ and $f_{b_{w}}(\cdot \mid X, I)$ is done via

$$
\widehat{F}_{b_{w}}\left(b_{w} \mid Z, I\right)=\frac{\frac{1}{L_{I} h_{Z}^{d}} \sum_{\ell=1}^{L_{I}} \mathbb{I}\left(I_{\ell}=I\right) \mathbb{I}\left(b_{w_{\ell}} \leq b_{w}\right) K\left(\frac{Z-Z_{\ell}}{h_{Z}}\right)}{\frac{1}{L_{I} h_{Z}^{d}} \sum_{\ell=1}^{L_{I}} \mathbb{I}\left(I_{\ell}=I\right) K\left(\frac{Z-Z_{\ell}}{h_{Z}}\right)}
$$

and

$$
\widehat{f}_{b_{w}}\left(b_{w} \mid Z, I\right)=\frac{\frac{1}{L_{I} h_{Z}^{d}} \sum_{\ell=1}^{L_{I}} \mathbb{I}\left(I_{\ell}=I\right) \mathbb{I}\left(b_{w_{\ell}} \leq b_{w}\right) K\left(\frac{Z-Z_{\ell}}{h_{Z}}\right)}{\frac{1}{L_{I} h_{Z}^{d}} \sum_{\ell=1}^{L_{I}} \mathbb{I}\left(I_{\ell}=I\right) K\left(\frac{Z-Z_{\ell}}{h_{Z}}\right)},
$$

where $K(u)$ is a kernel function and $h_{Z}$ a vanishing bandwidth. For the four experiments presented below, I consider $L=100$ auctions with $I=2$ bidders. The kernel function is an Epanechnikov, $K(u)=\frac{3}{4}\left(1-u^{2}\right) \mathbb{I}(|u| \leq 1)$, and the bandwidth choice follows Silverman's rule of thumb, $h_{j}^{*}=C_{q}(k, d) \widehat{\sigma}_{j} L^{-1 /(2 q+d)}$, where $C_{q}(k, p)$ is a constant that depends on the kernel order $(q=2)$, the kernel type $(k=$ Epanechnikov) and the number of covariates $(d)$; $\widehat{\sigma}_{j}$ is the standard deviation of the variable $Z_{j}$; and $L$ is the sample size. To investigate the effect of the bandwidth choice on the performance of the nonparametric estimator, five variations $^{11}$ of the optimal bandwidth $h_{j}^{*}$ are considered: $\left\{h_{j}^{*} / 3,2 h_{j}^{*} / 3, h_{j}^{*}, 4 h_{j}^{*} / 3,3 h_{j}^{*}\right\}$.

The performance of both estimation approaches is evaluated through the Root Mean Squared Error (RMSE)

$$
\operatorname{RMSE}[\hat{\theta}]=\sqrt{\mathbb{E}\left[(\widehat{\theta}-\theta)^{2}\right]},
$$

where in the equation above $\widehat{\theta}$ represents the corresponding estimate. Since the goal is to evaluate the performance of the quantile regression and the nonparametric methods in computing the optimal reservation price in each auction $\ell$, the RMSE is computed as

$$
\operatorname{RMSE}\left[\widehat{V}\left(\widehat{\alpha_{r}}\right)\right]=\sqrt{\frac{1}{S \times L} \sum_{s=1}^{S} \sum_{\ell=1}^{L}\left(\widehat{V}\left(\widehat{\alpha_{r_{s \ell}}} \mid X_{\ell}\right)-V\left(\alpha_{r_{s \ell}^{*}} \mid X_{\ell}\right)\right)^{2}}
$$

\footnotetext{
${ }^{11}$ Recall that a large bandwidth increases the estimation bias but reduces its estimated variance.
} 
where $V\left(\alpha_{r_{s \ell}^{*}} \mid X_{\ell}\right)$ is the true optimal reservation price at auction $\ell$ and $S$ is the total number of replications of the experiment. Therefore, the RMSE is evaluated over the number of auctions (sample size) and number of simulations. The four experiments are discussed below:

\section{Experiment 1: Univariate correctly specified model.}

Population Model: $V(\alpha \mid X)=\gamma_{0}(\alpha)+1 \times Z_{1}$, where

$$
\gamma_{0}(\alpha)=-\log \left(1-\left(1-\frac{1}{e}\right) \alpha\right)
$$

and $Z_{1} \sim \ln \mathcal{N}(0,0.5)$. The quantile function $\gamma_{0}(\alpha)$ is the one of an exponential distribution over $[0,1]$. In this first experiment, the estimated quantile regression model is correctly specified and the kernel function is a univariate kernel. The constant $C_{q}(k, d)$ $C_{2}($ Epanechnikov, 1$)=2.34$.

\section{Experiment 2: Bivariate correctly specified model.}

Population Model: $V(\alpha \mid X)=\gamma_{0}(\alpha)+1 \times Z_{1}+\gamma_{2}(\alpha) \times Z_{2}$, where $\gamma_{0}(\alpha)$ and $Z_{1}$ are as above,

$$
\gamma_{2}(\alpha)=1-\frac{1}{e^{\alpha}}
$$

and $Z_{2} \sim \operatorname{Exp}(\lambda=1)$. The slope coefficient $\gamma_{2}(\alpha)$ is such that bidders in the lower part of the distribution are not affected by the covariate $Z_{2}$, whereas this effect increases with $\alpha$. In this second experiment, the estimation considers a correctly specified model and because there are two covariates $Z=\left(Z_{1}, Z_{2}\right)$ the constant used in the bandwidth choice is $C_{2}$ (Epanechnikov, 2$)=2.20$. Experiments 1 and 2 evaluate the effect of an additional covariate on the estimation performance when the private value quantile function is correctly specified. 


\section{Experiment 3: Misspecified model ( $Z_{2}$ is omitted from the model).}

Population Model: $V(\alpha \mid X)=\gamma_{0}(\alpha)+1 \times Z_{1}+\gamma_{2}(\alpha) \times Z_{2}$.

Quantile Regression Estimated Model: $V(\alpha \mid X)=\gamma_{0}(\alpha)+1 \times Z_{1}$.

\section{Experiment 4: Misspecified model ( $Z_{1}$ is omitted from the model).}

Population Model: $V(\alpha \mid X)=\gamma_{0}(\alpha)+1 \times Z_{1}+\gamma_{2}(\alpha) \times Z_{2}$.

Quantile Regression Estimated Model: $V(\alpha \mid X)=\gamma_{0}(\alpha)+\gamma_{2}(\alpha) \times Z_{2}$.

\section{Experiment 5: Misspecified model (functional form).}

Population Model: $V(\alpha \mid X)=\gamma_{0}(\alpha)+1 \times Z_{1}+\gamma_{2}(\alpha) \times Z_{1}^{2}$.

Quantile Regression Estimated Model: $V(\alpha \mid X)=\gamma_{0}(\alpha)+1 \times Z_{1}$.

Experiments 3, 4 and 5 investigate the effect of misspecifications in the private value quantile function on the performance of both estimators. Experiment 3 considers a misspecified model in the estimation by omitting the covariate $Z_{2}$. Note that in the nonparametric estimation, a univariate kernel is used. Since the slope coefficient $\gamma_{2}(\alpha)$ attenuates the effect of $Z_{2}$ on the lower part of the private value distribution, the effect of an omitted variable on the estimation of the optimal reservation price (which very likely is in the lower part of the private value distribution) may be subestimated. For this reason, experiment 4 considers a misspecified model by omitting $Z_{1}$ from the model. Note that $\gamma_{1}(\alpha)=\gamma_{1}=1$, that is, changes in $Z_{1}$ affect all the quantiles of the private value distribution in the same way. Finally, experiment 5 considers misspecification in the quantile functional form. The number of covariates in both the population and the estimated models is the same (univariate), whereas the true model is nonlinear in $Z$.

The results of 1,000 replications of the five experiments described above are grouped on Table 1. The root mean squared errors of the nonparametric estimation are given on the second to sixth columns, whereas the one of the quantile regression is in the 7 th column. In 
Table 1

Results of the Monte Carlo Experiments

Root Mean Squared Error

\begin{tabular}{|c|c|c|c|c|c|c|}
\hline & \multicolumn{5}{|c|}{ Nonparametric } & \multirow[t]{2}{*}{ Quantile Regression } \\
\hline & $h_{j}^{*} / 3$ & $2 h_{j}^{*} / 3$ & $h_{j}^{*}$ & $4 h_{j}^{*} / 3$ & $3 h_{j}^{*}$ & \\
\hline Experiment 1 & 0.5061 & 0.4603 & 0.4373 & 0.4317 & 0.5824 & 0.0983 \\
\hline Experiment 2 & 0.9390 & 0.6851 & 0.5787 & 0.5366 & 0.6466 & 0.2399 \\
\hline Experiment 3 & 0.7792 & 0.6139 & 0.5328 & 0.5192 & 0.6590 & 0.3978 \\
\hline Experiment 4 & 1.0467 & 0.8500 & 0.7805 & 0.7326 & 0.7412 & 0.7037 \\
\hline Experiment 5 & 0.9680 & 0.8371 & 0.8001 & 0.7954 & 1.0153 & 0.3874 \\
\hline
\end{tabular}

bold are the smallest root mean squared error of the nonparametric estimation and the one computed using the quantile regression approach (which does not depend on a bandwidth choice). Note that in all the experiments, the quantile regression approach gives smaller (in most of the cases considerably smaller) root mean squared errors. A first remark is for experiment 4 , whose root mean squared error is very close to the minimium one of the nonparametric approach: although omitting an important variable deteriorates both estimation approaches, the quantile regression still gives a better result. A second remark is for experiment 5: it is expected that nonparametric approaches offer a better fit when dealing with misspecification in the functional form. However, the quantile regression approach still gives a better result. This suggest that the variance of the nonparametric estimator is large enough to dominate the misspecification bias of the quantile regression estimator.

\subsection{Asymptotic Properties of the Estimator}

In this section, the asymptotic properties of the private value quantile regression estimator (3.11) are studied. In what follows, let

$$
Q(\gamma \mid \alpha, I)=\mathbb{E}\left[\rho_{\Psi_{I}(\alpha)}\left(b_{w}-h(X \gamma)\right) \mid I\right]
$$




$$
\widehat{Q}(\gamma \mid \alpha, I)=\frac{1}{L_{I}} \sum_{\ell=1}^{L_{I}} \mathbb{I}\left(I_{\ell}=I\right) \rho_{\Psi_{I}(\alpha)}\left(b_{w_{\ell}}-h\left(X_{\ell} \gamma\right)\right)
$$

and

$$
\widehat{Q}(\widehat{\gamma} \mid \alpha, I)=\widehat{Q}(\widehat{\gamma}(\alpha \mid I) \mid \alpha, I)=\min _{\gamma \in \Gamma} \widehat{Q}(\gamma \mid \alpha, I)
$$

be the population, the empirical and the optimized quantile regression objective functions. The first and second derivatives of $Q(\gamma \mid \alpha, I)$ with respect to $\gamma$ will be denoted, respectively, by $Q_{\gamma}(\gamma \mid \alpha, I)$ and $Q_{\gamma \gamma}(\gamma \mid \alpha, I)$, whereas $h^{(1)}(\cdot)$ and $h^{(2)}(\cdot)$ are respectively the first and second derivatives of $h(\cdot)$.

In the case of a misspecified model, $\widehat{\gamma}(\alpha \mid I)$ is expected to converge to a pseudo-true private value quantile regression coefficient defined as

$$
\gamma^{*}(\alpha \mid I)=\arg \min _{\gamma \in \Gamma} Q(\gamma \mid \alpha, I)
$$

where the expectation is taken with respect to the true model distribution. In what follows, it is assumed that the considered pseudo-true values are uniquely defined.

Theorem 5 gives the asymptotic distribution of the private value quantile regression estimator (3.11). Its proof relies on arguments sketched in Newey and McFadden (1994) for estimators optimizing non smooth objective functions.

Theorem 5 Under assumptions 1-2, 4-6 and if $\gamma^{*}(\alpha \mid I)$ from (3.16) is an inner point of $\Gamma$,

$$
\sqrt{L_{I}}\left(\widehat{\gamma}(\alpha \mid I)-\gamma^{*}(\alpha \mid I)\right) \stackrel{d}{\longrightarrow} \mathcal{N}\left(0, Q_{\gamma \gamma}^{-1}\left(\gamma^{*} \mid \alpha, I\right) J\left(\gamma^{*} \mid \alpha, I\right) Q_{\gamma \gamma}^{-1}\left(\gamma^{*} \mid \alpha, I\right)\right),
$$

where

$$
\begin{gathered}
J\left(\gamma^{*} \mid \alpha, I\right)=\mathbb{E}\left[\left\{F_{b_{w}}\left(h\left(X \gamma^{*}(\alpha \mid I)\right) \mid X, I\right)-2 \Psi_{I}(\alpha) F_{b_{w}}\left(h\left(X \gamma^{*}(\alpha \mid I)\right) \mid X, I\right)\right.\right. \\
\left.\left.+\Psi_{I}(\alpha)^{2}\right\} h^{(1)}\left(X \gamma^{*}(\alpha \mid I)\right)^{2} X^{\prime} X \mid I\right]
\end{gathered}
$$


and

$$
\begin{aligned}
Q_{\gamma \gamma}\left(\gamma^{*} \mid \alpha, I\right)=\mathbb{E}\left[f_{b_{w}}\left(h\left(X \gamma^{*}(\alpha \mid I)\right) \mid X, I\right) h^{(1)}\left(X \gamma^{*}(\alpha \mid I)\right)^{2} X^{\prime} X \mid I\right]+ \\
\mathbb{E}\left[\left(F_{b_{w}}\left(h\left(X \gamma^{*}(\alpha \mid I)\right) \mid X, I\right)-\Psi_{I}(\alpha)\right) h^{(2)}\left(X \gamma^{*}(\alpha \mid I)\right) X^{\prime} X \mid I\right]
\end{aligned}
$$

$F_{b_{w}}(\cdot \mid X, I)=\Psi_{I}\left(F_{v}(\cdot \mid X, I)\right)$ and $f_{b_{w}}(\cdot \mid X, I)$ being the c.d.f. and p.d.f. of the winning bids given $(X, I)$.

Although $\gamma(\alpha \mid I)$ is a parameter of the private value distribution, the asymptotic variance of $\widehat{\gamma}(\alpha \mid I)$ in the Theorem is computed using the winning bids distribution. Note that if the model is correctly specified, then $\gamma^{*}(\alpha \mid I)=\gamma(\alpha \mid I)$ and $F_{b_{w}}\left(h\left(X \gamma^{*}(\alpha \mid I)\right) \mid X, I\right)=\Psi_{I}(\alpha)$, so that

$$
J(\gamma \mid \alpha, I)=\Psi_{I}(\alpha)\left(1-\Psi_{I}(\alpha)\right) \mathbb{E}\left[h^{(1)}(X \gamma(\alpha \mid I))^{2} X^{\prime} X \mid I\right]
$$

and

$$
Q_{\gamma \gamma}(\gamma \mid \alpha, I)=\mathbb{E}\left[f_{b_{w}}(h(X \gamma(\alpha \mid I)) \mid X, I) h^{(1)}(X \gamma(\alpha \mid I))^{2} X^{\prime} X \mid I\right]
$$

The asymptotic variance of the quantile regression estimator can be estimated using techniques described in Koenker (2005). The applications considered here uses bootstrap inference and for this reason the variance estimation aspects are not detailed.

If the private value distribution is independent upon $I$ given $X$, then

$$
\begin{aligned}
\gamma^{*}(\alpha \mid I) & =\arg \min _{\gamma \in \Gamma} \mathbb{E}\left[\rho_{\Psi_{I}(\alpha)}\left(b_{w}-h(X \gamma)\right) \mid I\right] \\
& =\arg \min _{\gamma \in \Gamma} \mathbb{E}\left[\rho_{\Psi_{I}(\alpha)}\left(b_{w}-h(X \gamma)\right)\right] \\
& =\gamma^{*}(\alpha),
\end{aligned}
$$

which opens space for a potential improvement in the estimation efficiency since samples 
with different number of bidders $I_{\ell}$ can be pooled, increasing the sample size to $L \geq L_{I}$. This therefore leads to consider

$$
\widehat{\gamma}(\alpha)=\arg \min _{\gamma \in \Gamma} \widehat{Q}(\gamma \mid \alpha)
$$

where

$$
\widehat{Q}(\gamma \mid \alpha)=\frac{1}{L} \sum_{\ell=1}^{L} \rho_{\Psi_{I_{\ell}}(\alpha)}\left(b_{w_{\ell}}-h\left(X_{\ell} \gamma\right)\right)
$$

Let

$$
\gamma^{*}(\alpha)=\arg \min _{\gamma \in \Gamma} Q(\gamma \mid \alpha) \text { where } Q(\gamma \mid \alpha)=\mathbb{E}\left[\rho_{\Psi_{I}(\alpha)}\left(b_{w}-h(X \gamma)\right)\right]
$$

The following Corollary gives the asymptotic distribution of the pooled quantile regression estimator.

Corollary 6 Under assumptions 1-2 and 4-6,

$$
\sqrt{L}\left(\widehat{\gamma}(\alpha)-\gamma^{*}(\alpha)\right) \stackrel{d}{\longrightarrow} \mathcal{N}\left(0, Q_{\gamma \gamma}^{-1}\left(\gamma^{*} \mid \alpha\right) J\left(\gamma^{*} \mid \alpha\right) Q_{\gamma \gamma}^{-1}\left(\gamma^{*} \mid \alpha\right)\right)
$$

where $\gamma^{*}(\alpha)=\arg \min _{\gamma \in \Gamma} Q(\gamma \mid \alpha)$, with $Q(\gamma \mid \alpha)=\mathbb{E}[\widehat{Q}(\gamma \mid \alpha)]$, is the pseudo-true private value quantile regression coefficient,

$$
\begin{aligned}
J\left(\gamma^{*} \mid \alpha\right)=\mathbb{E}\left[\left\{F_{b_{w}}(\right.\right. & \left.\left.\left(X \gamma^{*}(\alpha)\right) \mid X, I\right)-2 \Psi_{I}(\alpha) F_{b_{w}}\left(h\left(X \gamma^{*}(\alpha)\right) \mid X, I\right)+\Psi_{I}(\alpha)^{2}\right\} \\
& \left.\times h^{(1)}\left(X \gamma^{*}(\alpha)\right)^{2} X^{\prime} X\right]
\end{aligned}
$$

and

$$
\begin{aligned}
Q_{\gamma \gamma}\left(\gamma^{*} \mid \alpha\right)=\mathbb{E}\left[f_{b_{w}}\left(h\left(X \gamma^{*}(\alpha)\right) \mid X, I\right) h^{(1)}\left(X \gamma^{*}(\alpha)\right)^{2} X^{\prime} X\right]+ \\
\mathbb{E}\left[\left(F_{b_{w}}\left(h\left(X \gamma^{*}(\alpha)\right) \mid X, I\right)-\Psi_{I}(\alpha)\right) h^{(2)}\left(X \gamma^{*}(\alpha)\right) X^{\prime} X\right],
\end{aligned}
$$


$F_{b_{w}}(\cdot \mid X, I)=\Psi_{I}\left(F_{v}(\cdot \mid X)\right)$ and $f_{b_{w}}(\cdot \mid X, I)$ being the c.d.f. and p.d.f. of the winning bids given $(X, I)$.

Since $L$ can be substantially larger than $L_{I}$, Corollary 6 suggests that $\widehat{\gamma}(\alpha)$ can considerably improve on $\widehat{\gamma}(\alpha \mid I)$ when the private values distribution is independent from $I$ given $X$.

The regression specification of Rezende $(2008)^{12}$ can offer another interesting source of improvement under special conditions. Assume for instance that, for $h(\cdot)$ as in Assumption 6 ,

$$
v_{i \ell}=h\left(\gamma_{0}+Z_{\ell} \gamma_{1}+\varepsilon_{i \ell}\right)
$$

where the $\varepsilon_{i \ell}$ are i.i.d. and independent of $\left(Z_{\ell}, I_{\ell}\right)$. In this case the conditional quantile function of the private values is

$$
V(\alpha \mid Z)=h\left(\gamma_{0}(\alpha)+Z \gamma_{1}\right) \text { where } \gamma_{0}(\alpha)=\gamma_{0}+F_{\varepsilon}^{-1}(\alpha)
$$

For such specification, pooling over $\alpha$ can improve the estimation of the slope coefficients $\gamma_{1}$ as proposed by Zou and Yuan (2008) with the Composite Quantile Regression (CQR) estimator

$$
\widehat{\gamma}_{\mathrm{CQR}}=\arg \min _{\gamma_{0,1}, \ldots, \gamma_{0, K}, \gamma_{1}} \frac{1}{K} \sum_{k=1}^{K}\left\{\frac{1}{L} \sum_{\ell=1}^{L} \rho_{\Psi_{I_{\ell}}\left(\alpha_{k}\right)}\left(b_{w_{\ell}}-h\left(\gamma_{0, k}+Z_{\ell} \gamma_{1}\right)\right)\right\}
$$

where $\widehat{\gamma}_{\mathrm{CQR}}=\left(\widehat{\gamma}_{0}\left(\alpha_{1}\right), \ldots, \widehat{\gamma}_{0}\left(\alpha_{K}\right), \widehat{\gamma}_{1}\right)^{\prime}$ and $\alpha_{k} \in[0,1], k=1, \ldots, K$, are some quantile levels. Zou and Yuan (2008) have shown in particular that the CQR estimator can improve on least squares estimation. That estimator is asymptotically normal with a limit variance that can be derived with arguments similar to the ones used in Theorem 5. However, implementing this strategy with the considered auction dataset has led to a rejection of the correct specification of (3.19) (that is, a rejection of $\gamma_{1}(\alpha)=\gamma_{1}$ for all retained $\alpha_{k}$ ) so that

\footnotetext{
${ }^{12}$ For simplicity of notation, it is assumed independence of the private value distribution upon $I$.
} 
the limit distribution of the CQR estimator is not detailed here for the sake of brevity.

\section{Auction Hypotheses Testing}

The estimation methodology developed above enables us to investigate some interesting questions for the empirical auction literature that are grouped in the following three hypotheses tests: exclusion participation restriction, functional form of the private values conditional quantile function and constancy in the impact of the auction characteristics across the private values distribution. In what follows, $\mathcal{A}=\left\{\alpha_{1}, \cdots, \alpha_{K}\right\}$ is a set of prescribed quantile levels used in the considered test statistics.

\subsection{Testing the Exclusion Participation Restriction}

As discussed in section 2.1, testing the condition $V(\cdot \mid X, I)=V(\cdot \mid X)$ gives indication about potential unobserved heterogeneity or presence of a entry stage in the auction game. An important policy implication of that condition is that the optimal reservation price is also not dependent upon the number of actual bidders $I$ as usual in the auction literature. In addition, as discussed in the previous section, the exclusion participation restriction enables a potential improvement in the estimation efficiency. The considered null and alternative hypotheses are

$$
\begin{aligned}
& H_{0}: \gamma^{*}(\alpha \mid I)=\gamma^{*}(\alpha) \text { for all } \alpha \in \mathcal{A} \text { and } I \in \mathcal{I} \\
& H_{1}: \operatorname{not} H_{0} .
\end{aligned}
$$

Note that even if $V(\cdot \mid X, I)$ differs from $h(X \gamma(\cdot \mid I))$, i.e. the function $h(\cdot)$ is misspecified, the pseudo-true coefficients still satisfy $\gamma^{*}(\cdot \mid I)=\gamma^{*}(\cdot)$ when $V(\cdot \mid X, I)=V(\cdot \mid X)$.

A simple way of testing the null hypothesis above is via a Wald test, jointly for all 
the coefficients and quantile levels. However, the latter involves standardization of the test-statistic by the variance-covariance matrix of the coefficients, which in turn involves estimation of the unknown density function of the random errors. The estimation of the latter requires either a bandwidth choice (see Powell (1991)) or bootstrap resampling methods (see Buchinsky (1995)). Some preliminary experiments had suggested that an alternative strategy as described below may give better results.

A strategy similar to a maximum likelihood ratio test can be implemented to avoid the estimation of the variance-covariance matrix. Let $\widehat{Q}(\widehat{\gamma} \mid \alpha)$ represents the optimized pooled objective function, i.e.

$$
\widehat{Q}(\widehat{\gamma} \mid \alpha)=\widehat{Q}(\widehat{\gamma}(\alpha) \mid \alpha)=\min _{\gamma \in \Gamma} \widehat{Q}(\gamma \mid \alpha)
$$

and $\widehat{Q}(\widehat{\gamma} \mid \alpha, I)$ the optimized individual objective function as defined in (3.15). Under the null hypothesis of independence, $Q(\gamma \mid \alpha)=\sum_{I \in \mathcal{I}} Q(\gamma \mid \alpha, I) \mathbb{P}(I)$. This leads to consider the distance metric statistics in the terminology of Newey and McFadden (1994), or the M-statistic in the terminology of Rao and Zhao (1992),

$$
M_{\text {Ind }}=\widehat{Q}(\widehat{\gamma} \mid \alpha)-\sum_{I \in \mathcal{I}} \widehat{Q}(\widehat{\gamma} \mid \alpha, I) L_{I} / L
$$

In the application, the critical values and p-values of tests based on (4.21) will be calculated using the random weighting bootstrap method proposed by Rao and Zhao (1992), Wang and Zhou (2004) and Zhao, Wu and Yang (2007), which is detailed in the Appendix B. Appendix C describes a Monte Carlo experiment of the test of exclusion participation restriction and its corresponding rejection probabilities. 


\subsection{Choice of Specification for the Bidders' Private Values: Linear versus Exponential}

As mentioned earlier, the interest here is to choose between a linear or exponential function for the quantile regression specification (2.4). While the linear specification is apparently more popular and simpler to estimate, the exponential specification delivers positive private values, which may not be the case of a linear one. Both models may be also misspecified for the data at hand. An additional difficulty is that the retained specification must be valid for several quantile levels. This section thus proposes a test to investigate which of the two model specifications has a better measure of fit for a prescribed range of quantiles.

There is a extensive literature on testing hypotheses for model selection and goodness of fit. See e.g. Koenker and Machado (1999) for nested quantile regression models, White (1982) for conditional mean analysis, Zheng (1998), Horowitz and Spokoiny (2001) and Whang (2006) for parametric against nonparametrics alternatives.

Considering model selection and non-nested hypothesis testing, Vuong (1989) has proposed likelihood ratio tests using the Kullback-Leibler (1951) information criterion, which measures the distance between a given distribution and the true distribution function. The best model among a collection of competing models is defined to be the one that is closest to the true distribution. The tests are derived for cases in which the models are non-nested, overlapping, or nested and whether both, one, or neither is misspecified. The null hypothesis is the equivalence of the two specifications, i.e. the two specifications are at the same distance of the true distribution function, although dominance can also be considered.

Based on the insights of Vuong (1989), the test proposed in this section leads to compare the population objective function measures of the linear and the exponential models. Let 
the population objective functions under the previous two models be defined respectively as

$$
Q_{\mathrm{L}}(\gamma \mid \alpha, I)=\mathbb{E}\left[\rho_{\Psi_{I}(\alpha)}\left(b_{w}-X \gamma\right) \mid I\right]
$$

and

$$
Q_{\mathrm{E}}(\gamma \mid \alpha, I)=\mathbb{E}\left[\rho_{\Psi_{I}(\alpha)}\left(b_{w}-\exp (X \gamma)\right) \mid I\right]
$$

Define the pseudo-true private values quantile regression coefficients as $\gamma_{j}^{*}(\alpha \mid I)=\arg \min _{\gamma \in \Gamma} Q_{j}(\gamma \mid \alpha, I)$ and the infimum of the population objective functions as

$$
Q_{j}\left(\gamma_{j}^{*} \mid \alpha, I\right)=Q_{j}\left(\gamma_{j}^{*}(\alpha \mid I) \mid \alpha, I\right)=\inf _{\gamma \in \Gamma} Q_{j}(\gamma \mid \alpha, I)
$$

where $j=$ E,L.

The next set of hypotheses considers dominance of the exponential specification:

$$
\begin{aligned}
& H_{0}: Q_{\mathrm{E}}\left(\gamma_{\mathrm{E}}^{*} \mid \alpha, I\right)-Q_{\mathrm{L}}\left(\gamma_{\mathrm{L}}^{*} \mid \alpha, I\right) \leq 0 \text { for all } \alpha \in \mathcal{A} \text { and } I \in \mathcal{I} \\
& H_{1}: \operatorname{not} H_{0},
\end{aligned}
$$

which in turn motivates the following test statistic:

$$
\widehat{\text { Vuong }}=\sqrt{L_{I}} \sup _{\alpha \in \mathcal{A}}\left(\widehat{Q}_{\mathrm{E}}\left(\widehat{\gamma}_{\mathrm{E}} \mid \alpha, I\right)-\widehat{Q}_{\mathrm{L}}\left(\widehat{\gamma}_{\mathrm{L}} \mid \alpha, I\right)\right)
$$

Note that exchanging $\widehat{Q}_{\mathrm{E}}\left(\widehat{\gamma}_{\mathrm{E}} \mid \alpha, I\right)$ and $\widehat{Q}_{\mathrm{L}}\left(\widehat{\gamma}_{\mathrm{L}} \mid \alpha, I\right)$ in (4.22) gives a test statistic for dominance of the linear specification.

In the application, the critical values and p-values of the test based on (4.22) will be computed by the pairwise bootstrap method in each original subsample $L_{I}{ }^{13}$, i.e. samples of

\footnotetext{
${ }^{13}$ This form of bootstrap is also known as $(\mathrm{X}, \mathrm{Y})$-pair bootstrap method and has been widely used in the quantile regression literature.
} 
the $\left(X_{\ell}, b_{w_{\ell}}\right)$ pairs are drawn with replacement from the $L_{I}$ pairs $\left\{\left(X_{\ell}, b_{w_{\ell}}\right): \ell=1, \cdots, L_{I}\right\}$ of the original subsample, each with probability $1 / L_{I}$. Appendix $\mathrm{C}$ describes a Monte Carlo experiment of the specification test above and its rejection probabilities.

\subsection{Constancy of the Slope Coefficients}

In this section, the impact of changes in the auctioned good characteristics across the entire distribution of private values is investigated. Consider the private values conditional quantile specification given in (2.3), where the vector $\gamma_{1}(\alpha \mid I)$ groups all the slope coefficients, excluding so the intercept. The hypothesis of interest is that $\gamma_{1}(\alpha \mid I)$ does not depend upon $\alpha$, in which case the model (2.3) with $h(t)=t$ is the regression model of Rezende (2008).

The null and alternative hypothesis considered in this test are therefore:

$$
\begin{aligned}
& H_{0}: \gamma_{1}^{*}(\alpha \mid I)=\gamma_{1}^{*}(I) \text { for all } \alpha \in \mathcal{A} \text { and } I \in \mathcal{I} \\
& H_{1}: \operatorname{not} H_{0} .
\end{aligned}
$$

Define the CQR empirical objective function as

$$
\widehat{Q}_{\mathrm{CQR}}\left(\gamma_{\mathrm{CQR}} \mid I\right)=\frac{1}{K} \sum_{k=1}^{K}\left\{\frac{1}{L} \sum_{\ell=1}^{L} \rho_{\Psi_{I_{\ell}}\left(\alpha_{k}\right)}\left(b_{w_{\ell}}-h\left(\gamma_{0, k}+Z_{\ell} \gamma_{1}\right)\right)\right\} .
$$

Under the null hypothesis, the CQR population objective function equals the average of the individual objective functions over the set of prescribed quantiles $\mathcal{A}=\left\{\alpha_{1}, \cdots, \alpha_{K}\right\}$. The test statistic proposed here will be constructed as in the test of exclusion participation restriction by using the M-statistic defined as follows

$$
M_{\mathrm{CQR}}=L\left[\widehat{Q}_{\mathrm{CQR}}\left(\widehat{\gamma}_{\mathrm{CQR}} \mid I\right)-\frac{1}{K} \sum_{k=1}^{K} \widehat{Q}\left(\widehat{\gamma} \mid \alpha_{k}, I\right)\right]
$$


where $\widehat{Q}_{\mathrm{CQR}}\left(\widehat{\gamma}_{\mathrm{CQR}} \mid I\right)$ is the optimized CQR objective function.

The critical values and p-values of tests based on (4.23) will be calculated using the random weighting bootstrap method as described in the Appendix B. A Monte Carlo experiment for the test of constancy of slope coefficients above and the corresponding rejection probabilities are given in the Appendix C.

\section{Empirical Application}

In this section, I illustrate empirically the methodology proposed in the paper using data from ascending timber auctions run by the USFS. Timber auctions data have been used in several empirical studies, see e.g. Baldwin, Marshall and Richard (1997), Haile (2001), Athey and Levin (2001), Athey, Levin and Seira (2011), Li and Zheng (2012), AradillasLopez, Gandhi and Quint (2013), Li and Perrigne (2003) and others. Some other works have investigated risk-aversion on timber auctions, as e.g. Lu and Perrigne (2008), Athey and Levin (2001) and Campo, Guerre, Perrigne and Vuong (2011).

\subsection{The Timber Auction Data}

The dataset used here is publicly available on the internet ${ }^{14}$. It aggregates ascending auctions from the states covering the western half of the US (regions 1-6 as labeled by the USFS) occurred in 1979. It contains 472 auctions involving a total of 1175 bids and a set of variables characterizing each timber tract including the estimated volume of the timber measured in thousand of board feet (or mbf) and its estimated appraisal value given in Dollar per unit of volume. The latter measures how much the tract worths at the present time, taking into consideration a combination of factors as volume of each specie, quality and marketable

\footnotetext{
${ }^{14}$ The same dataset was used by Haile and Tamer (2003), Lu and Perrigne (2008) and Aradillas-Lopez et al. (2013), and it is available at the JAE Data Archieve website: http://qed.econ.queensu.ca/jae/2008v23.7/lu-perrigne/
} 
price. Only scaled sales are considered, in which bids are per unit of timber. Therefore, winning bids are given in Dollar per unit of volume.

The government conducts a cruise of the timber tract prior to the auction and publishes a report with the characteristics of the tract uncovered by the cruise. The reservation price is announced prior to the auction and equals the appraisal value of the $\operatorname{tract}^{15}$. The auction is conducted in two rounds: in the first round, bidders submit sealed bids ${ }^{16}$ that must exceed the appraisal value of the tract to be qualified for the auction; in the second round, bidders compete in an ascending auction.

Table 2

Summary Statistics

\begin{tabular}{lrrrr}
\hline \hline & Winning Bids & Appraisal Value & Volume & Number of Bidders \\
\hline Mean & 129.17 & 72.34 & $3,171.10$ & 2.49 \\
Std. Deviation & 119.76 & 52.92 & $4,418.20$ & 0.50 \\
$25 \%$ & 59.13 & 24.47 & 294 & 2 \\
$50 \%$ & 113.81 & 68.38 & 967 & 2 \\
$75 \%$ & 172.53 & 111.25 & 4,724 & 3 \\
Min & 0.30 & 0.25 & 12 & 2 \\
Max & $1,981.50$ & 219.58 & 24,800 & 3 \\
Skewness & 8.16 & 0.44 & 1.99 & 0.04 \\
Observations & 472 & 472 & 472 & 472 \\
\hline
\end{tabular}

Table 2 gives some descriptive statistics about the dataset. The auctioned tract displays significant heterogeneity in quality and size. The mean of the variables are all above the median, indicating tailored marginal distributions. In particular, the marginal distribution of winning bids is highly positive skewed. In the dataset, the number of actual bidders $I$ takes values 2 and 3, with $I=2$ more than $50 \%$ of the cases. Table 3 provides the results of a median regression analysis of the winning bids on the timber appraisal value per mbf and the volume. Lu and Perrigne (2008) performed a mean regression analysis and

\footnotetext{
${ }^{15}$ It is well known that the screening effect of the appraisal value in timber auctions is almost negligible, being plausible to consider that the reservation price is nonbinding. See e.g. Campo, Guerre, Perrigne and Vuong (2011), Haile and Tamer (2003) and Aradillas-Lopez at al. (2013).

${ }^{16}$ Note that the bids in the first round are not proper bids, but a proposal to be qualified for bidding in the second round.
} 
concluded that the estimated appraisal value and the volume of the timber are the variables that better explain the winning bid's variability. Both variables are also highly significant in the median analysis, which can be seen by the coefficient of determination $R_{\alpha}$ at the median quantile $^{17}$. For this reason, I consider a two-dimensional vector of covariates $Z$ grouping both the appraisal value per mbf and the volume of the timber ${ }^{18}$.

Table 3

OLS Estimation

\begin{tabular}{|c|c|c|c|c|}
\hline $\begin{array}{l}\text { Dependent Variable: Winning Bids } \\
\text { Regressors }\end{array}$ & Coefficient & Std. Deviation & t-value & p-value \\
\hline Intercept & $11.1265^{* * *}$ & 3.8554 & 2.886 & $4.08 \mathrm{E}-03$ \\
\hline Appraisal Value & $1.2873^{* * *}$ & 0.0505 & 25.514 & 0.000 \\
\hline Volume & $0.0029^{* * *}$ & 0.0008 & 3.503 & 0.0005 \\
\hline$R_{0.5}$ & 0.4159 & & & \\
\hline
\end{tabular}

\subsection{Results}

The choice of the set of prescribed quantiles $\mathcal{A}=\left\{\alpha_{1}, \cdots, \alpha_{K}\right\}$ used in the tests is an important issue. It would be ideal to estimate all the quantiles of the private values conditional distribution. However, as noticed by Koenker (2005) and also seen from our estimation results, the asymptotic precision of the quantile estimates in general, and the quantile regression estimates in particular, depend on the quantile density function evaluated at the quantile of interest. If the data are sparse at the quantile of interest, then the quantile

${ }^{17}$ The coefficient of determination is given by

$$
R_{\alpha}=1-\frac{\widehat{Q}\left(\widehat{\beta_{0}}, \widehat{\beta_{1}}, \widehat{\beta_{2}} \mid \alpha\right)}{\widehat{Q}\left(\widehat{\beta_{0}} \mid \alpha\right)},
$$

and represents how much of the variability in the winning bids is explained by both covariates above at the quantile level $\alpha$.

${ }^{18}$ Nonlinearity with respect to the appraisal value and the volume of the timber were also investigated. However, the results were not statistically significant. 
regression estimates will be less precise. This lack of precision was observed when estimating the quantiles closer to the boundary, leading to restrict the grid of quantile levels to $\mathcal{A}=\{0.12,0.14, \cdots, 0.80\}$. Another explanation for the lack of precision of the estimator close to the boundaries $\alpha=\{0,1\}$ is a technical feature of the quantile regression objective function. Since $\rho_{0}(u)=0$ for all $u \geq 0$, the objective function is zero for all $\beta$ such that $b_{w_{\ell}} \geq h\left(X_{\ell} \beta\right)$, for all $\ell$, leading to multiple minimizers. The same happens for $\alpha=1$. Gimenes and Guerre (2014) solve ${ }^{19}$ this issue by smoothing the quantile regression objective function, so that it becomes strictly convex for all $\alpha \in[0,1]$.

The first sealed bid auction stage used to qualify bidders for the ascending auction may select a certain number of bidders for the ascending auction stage, so that it might be interesting to use $V(\cdot \mid X, I)$, which depends upon I, in the policy analysis to be conducted. Roberts and Sweeting (2012) found evidences for a selective entry when studying timber auctions in California. They consider in the model a significant entry cost affecting bidders participation because bidders are allowed to conduct their own cruise. They argue that conducting a private cruise responds for a large fraction of the bidder's entry cost. Because bidders in our application do not conduct their own cruise, the entry cost might be small compared to the Californian timber auctions. There are nevertheless other costs that may affect bidders participation, such as developing a market studying, preparing the bids and attending to the auction. If indeed the entry costs are not relevant for the bidders' decision in participating, then the optimal reservation price policy could be chosen independently of $I$, as shown in Proposition 4-(ii).

Testing the relevance of a private value quantile function conditional upon bidder's participation can thus be very useful in the analysis that follows. Table 4 gives the results of the test of exclusion participation restriction suggested in section 4.1. There is not enough sta-

\footnotetext{
${ }^{19}$ Gimenes and Guerre (2014)'s approach can be easily extended to fit the ascending auction setup considered in this paper.
} 
Table 4

Test of the Exclusion Participation Restriction

\begin{tabular}{lccc}
\hline \hline Null Hypothesis & Specification & M-Statistic & p-value $^{\mathrm{a}}, \mathrm{b}$ \\
\hline \multirow{2}{*}{$\gamma^{*}(\alpha \mid I)=\gamma^{*}(\alpha)$ for all $\alpha \in \mathcal{A}$ and $I \in \mathcal{I}$} & Linear & 653.83 & 0.3096 \\
& Exponential & 720.37 & 0.4136 \\
\hline
\end{tabular}

a The p-value and critical values are computed using the random weighting bootstrap method. More details about the test are given in section 4.1;

$\mathrm{b}$ The number of bootstrapping replications is 5,$000 ; \mathcal{A}=\{0.12,0.14, \cdots, 0.80\}$ and $\mathcal{I}=\{2,3\}$.

tistical evidences to reject the null hypothesis of independence in both specification models (linear and exponential), implying that a possible improvement in efficiency can be achieved by pooling both samples. In other words, it is possible to rule out unobserved heterogeneity and entry stage affecting bidders participation. Note that an entry model as Levin and Smith (1994), where bidders do not know their private values at the entry stage, would generate private values independent upon $I$ as observed in Table 4. However, the bidders' participation decision in Levin and Smith (1994) is random, which may be considered a bit unrealistic.

Table 5 gives the results of the choice of specification test described in section 4 that investigates which of both regression specifications, linear or exponential, better represents the private value conditional quantiles. In the first row of Table 5, the test considers a null hypothesis in favour of the exponential specification, whereas in the second row the null hypothesis is reversed in favour of the linear specification. Both tests conclude that the linear specification dominates the exponential one over the set of prescribed quantiles $\mathcal{A}$. Therefore the linear specification is used in the rest of this section.

Table 6 provides the result of testing constancy of the slope coefficients. This test investigates whether changes in the auctioned characteristic $Z$ affect the private values quantiles similarly across the entire distribution. It may also show how heterogeneous ${ }^{20}$ are the bidders across the population. The test gives strong statistical evidence to reject the null hypothesis

\footnotetext{
${ }^{20}$ Recall that heterogeneity is the term used in this paper to represent the different reaction of the bidders to changes in the auctioned characteristics according to their rank in the private values conditional distribution.
} 
Table 5

Choice of Specification

\begin{tabular}{|c|c|c|}
\hline Null Hypothesis & Test Statistic & p-value ${ }^{a, b}$ \\
\hline$Q_{\mathrm{E}}\left(\gamma_{\mathrm{E}}^{*} \mid \alpha\right)-Q_{\mathrm{L}}\left(\gamma_{\mathrm{L}}^{*} \mid \alpha\right) \leq 0$ for all $\alpha \in \mathcal{A}$ & $20,142.55$ & $\overline{0.0000}$ \\
\hline$Q_{\mathrm{L}}\left(\gamma_{\mathrm{L}}^{*} \mid \alpha\right)-Q_{\mathrm{E}}\left(\gamma_{\mathrm{E}}^{*} \mid \alpha\right) \leq 0$ for all $\alpha \in \mathcal{A}$ & $-3,103.58$ & 0.9938 \\
\hline \multicolumn{3}{|c|}{$\begin{array}{l}\text { a The p-value and critical values are computed by resampling with replacement the } \\
\left(X_{\ell}, b_{w_{\ell}}\right) \text {-pair in each original subsample } L_{I} \text {. Note however that the result of in- } \\
\text { dependence given by the test of exclusion participation restriction is considered, so } \\
\text { that both samples with } I=\{2,3\} \text { are pooled in this analysis. More details about } \\
\text { the test are given in section } 4.2 \text {; } \\
\text { b The number of bootstrapping replications is } 5,000 ; \mathcal{A}=\{0.12,0.14, \cdots, 0.80\} \text {. }\end{array}$} \\
\hline
\end{tabular}

that the slope coefficients are constant across the quantiles $\alpha \in \mathcal{A}$. Therefore, bidders react differently to changes in the quality and size of the timber tract, as also clearly illustrated by Table 7 and Figures 1 and 2 below. Given the test result, the pooled quantile regression estimator provides a better characterization of the private value conditional quantiles than the CQR estimator defined in (3.20) and the mean analysis suggested by Rezende (2008).

Table 6

Constancy of the Slope Coefficients

\begin{tabular}{|c|c|c|}
\hline Null Hypothesis & M-Statistic & p-value ${ }^{a}, b$ \\
\hline$\gamma_{1}^{*}(\alpha)=\gamma_{1}^{*}$ for all $\alpha \in \mathcal{A}$ & 124.51 & 0.0048 \\
\hline \multicolumn{3}{|c|}{$\begin{array}{l}\text { The p-value and critical values are computed using the ran- } \\
\text { dom weighting bootstrap method. Note however that the } \\
\text { results obtained in the previous two tests were considered for } \\
\text { this analysis. More details about the test are given in section } \\
4.3 \text {; } \\
\text { The number of bootstrapping replications is } 5,000 ; \mathcal{A}= \\
\{0.12,0.14, \cdots, 0.80\} \text {. }\end{array}$} \\
\hline
\end{tabular}

The next Table and two following Figures describe the private values quantile regression coefficients. The most important variable is the appraisal value, a quality measure released by the seller, which is often interpreted as the seller's private value, see Lu and Perrigne (2008) and Aradillas-Lopez et al. (2013). The associated quantile regression coefficient is given in the second column of Table 7 and Figure 1 for a median auction, where the volume is 967 thousand of board feet and the appraisal value is about $\$ 68$ per thousand of board feet. Note that the coefficient is always significant and larger than 1, suggesting that it 
acts as a markup indicating how much more the auctioned good appraisal value is valued by the bidders than the seller. The private values can be also interpreted as a measure of how much the bidders would be willing to sell goods made with the timber bought at the auction ${ }^{21}$. This suggests that the higher the bidder's private value, the higher is his efficiency in aggregating value to the timber. The coefficients increase over the quantile levels, suggesting a relative increase in the markup of $75 \%$ when comparing bidders in the quantiles $\alpha=0.10$ and $\alpha=0.80$ of the private value conditional distribution. This is also evidence that bidders belonging to the upper tail of the private value distribution are more highly affected by changes in the appraisal value than median bidders.

Figure 1 shows the quantile regression and the OLS estimates of the appraisal value slopes with their corresponding $95 \%$ confidence intervals. Observe how the estimated markup increases over the quantile levels. Figure 2 shows the quantile regression and OLS estimates associated with the variable volume and their $95 \%$ confidence intervals. Although the coefficients seem to increase with the quantile level in Table 7, they lie inside the estimated OLS 95\% confidence intervals, suggesting that the volume coefficient may not depend upon $\alpha$. In the upper tail of the distribution, the coefficients are larger, but the confidence intervals are also wider.

In what follows $X(\tau)=\left(X_{1}(\tau), X_{2}(\tau), X_{3}(\tau)\right)$, where $X_{j}(\tau)$ is the quantile of order $\tau$ of the variable $X_{j}, j=1,2,3$. With some abuse of terminology, $X(\tau)$ will be called the quantile of order $\tau$ of the matrix of auction covariates $X, X(0.50)$ being called its median. Figure 3 gives the private value conditional quantile estimates for a median auction and their 95\% confidence intervals. Figure 4 presents the quantile estimates for several quantile levels of $X(\tau)$, where $\tau=\{0.15,0.25,0.50,0.75,0.85\}$. In particular, it shows the change in the shape of the private value quantiles due to variations in the quality and size of the timber

\footnotetext{
${ }^{21}$ In this interpretation, it is necessary to assume that timber is the most important component of the goods produced by the bidders. This could be however modified to cover other cases where timber would only be a part of these goods.
} 
Table 7

Private Value Quantile Regression Estimates

\begin{tabular}{cccc}
\hline \hline Quantile Level & Intercept & Appraisal Value & Volume \\
\hline 0.1 & 0.95 & 1.01 & 0.0007 \\
& {$[-0.97,2.28]$} & {$[0.99,1.04]$} & {$[0.0004,0.0016]$} \\
0.2 & 3.00 & 1.04 & 0.0016 \\
& {$[-0.72,8.49]$} & {$[0.99,1.13]$} & {$[0.0005,0.0027]$} \\
0.3 & 9.39 & 1.15 & 0.0018 \\
& {$[2.05,15.01]$} & {$[1.05,1.22]$} & {$[0.0010,0.0033]$} \\
0.4 & 11.77 & 1.25 & 0.0034 \\
& {$[5.32,20.83]$} & {$[1.14,1.33]$} & {$[0.0013,0.0049]$} \\
0.5 & 21.03 & 1.29 & 0.0041 \\
& {$[10.92,29.24]$} & {$[1.22,1.43]$} & {$[0.0023,0.0054]$} \\
0.6 & 35.68 & 1.36 & 0.0041 \\
& {$[21.63,45.02]$} & {$[1.27,1.56]$} & {$[0.0029,0.0055]$} \\
0.7 & 44.28 & 1.57 & 0.0045 \\
& {$[29.94,77.15]$} & {$[1.22,1.81]$} & {$[0.0029,0.0071]$} \\
0.8 & 67.64 & 1.75 & 0.0060 \\
& {$[32.91,101.02]$} & {$[1.31,2.02]$} & {$[0.0024,0.0138]$} \\
$0.9^{*}$ & 72.98 & 2.37 & 0.0167 \\
& {$[12.89,124.22]$} & {$[1.54,4.56]$} & {$[0.0031,0.0384]$}
\end{tabular}

The estimates are for a median auction and were computed using the pooled quantile regression estimator defined in (3.17). The $95 \%$ confidence interval of the quantile regression estimates in square brackets were computed by resampling with replacement the $\left(X_{\ell}, b_{w_{\ell}}\right)$-pair in each original subsample $L_{I}$;

* Note the loss in precision when $\alpha$ gets closer to the upper boundary. This is why such higher quantile have been excluded from the test statistics.

tract.

Figure 4 indeed shows that the auction covariates change significantly the shape of the private value distribution. This effect becomes even clear when comparing a high and a low quantile of the private value conditional distribution. Consider in particular the quantiles $\alpha=0.12$ and $\alpha=0.80$ of the private value conditional quantile curves and the quantiles of order $\tau=0.15,0.50$ and 0.85 of the auction covariates $X$, that is, auctions with low, median and high quality and size. The relative increase in the private value is of about $600 \%$ in the 
Figure 1

Appraisal Value Slope Coefficients

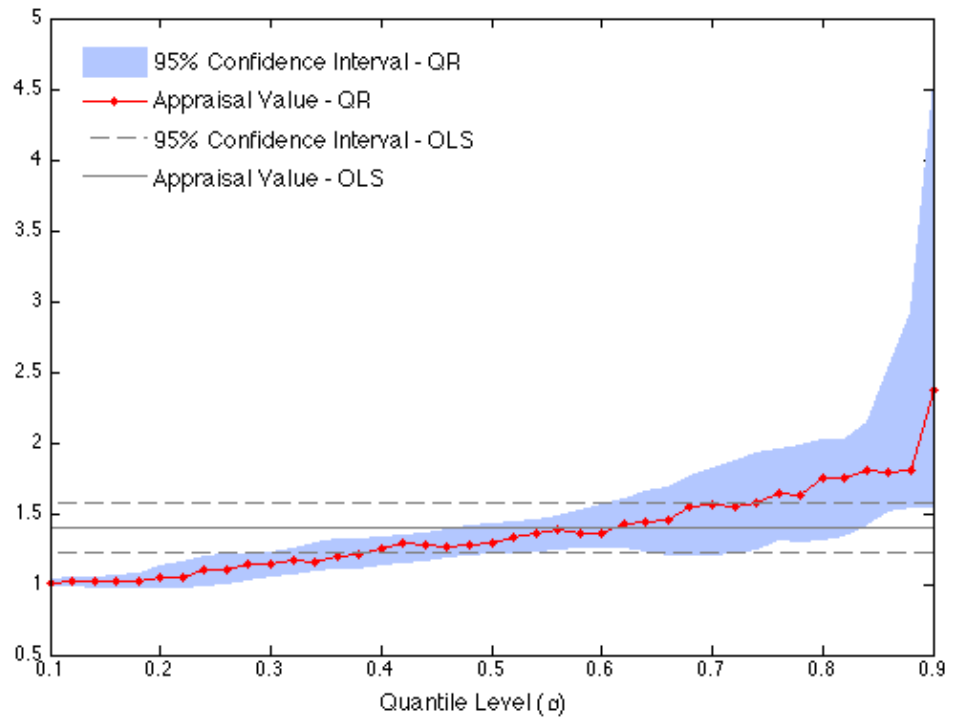

Notes: The $95 \%$ confidence intervals for the OLS estimate consider the heteroscedasticity-robust (White) standard errors. The ones for the quantile regression estimates were computed by resampling with replacement the $\left(X_{\ell}, b_{w_{\ell}}\right)$-pair in each original subsample $L_{I}$.

auctions with low quality and size, whereas it reduces to $172 \%$ and $142 \%$ in the median and high quality and size auctions, respectively. It seems that less productive bidders, i.e. the ones with a low rank $\alpha$, choose not to participate in auctions with higher quality and $\operatorname{size}^{22}$.

We now turn to the estimation of the seller's expected payoff and the associated optimal reservation price. The expected payoff of a seller with private values $v_{0}, \Pi\left(\alpha_{r} \mid X, v_{0}\right)$, is estimated using numerical integration via a trapezoidal rule to approximate the definite integral given in Proposition 4-(i). Figures 5 and 6 give the expected payoff of the seller for a median auction with the $95 \%$ confidence intervals for $I=2$ and $I=3$ bidders, respectively. In both Figures, the estimation procedures given by the pooled and the individual estimators defined respectively in (3.17) and (3.11) are compared. Observe that the former gives smaller

\footnotetext{
${ }^{22}$ Note that this suggests that the rank of the participant and the number of participants are simultaneously determined, so that quantile regression estimation can be affected by endogeneity. Addressing this issue is outside the scope of this paper.
} 
Figure 2: Volume Slope Coefficients

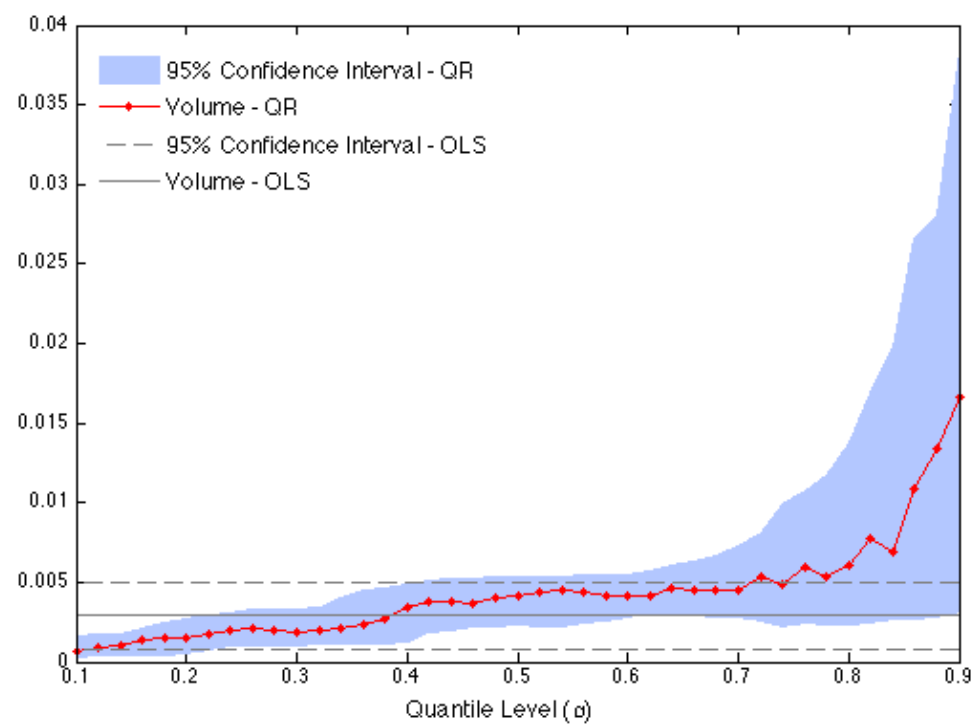

Notes: The 95\% confidence intervals for the OLS estimate consider the heteroscedasticity-robust (White) standard errors. The ones for the quantile regression estimates were computed by resampling with replacement the $\left(X_{\ell}, b_{w_{\ell}}\right)$-pair in each original subsample $L_{I}$.

confidence intervals than the latter due to its higher estimation efficiency. Note that the only difference between the seller's expected payoffs estimated via (3.17) and (3.11) is given by the way the private value conditional quantiles are estimated. In the former, both subsamples with different number of bidders are pooled, whereas in the latter the estimation is done for each $I$.

The sensitivity of the seller's expected payoff and the associated optimal reservation price to choices of the seller's private value $v_{0}$ will be now investigated. The choice of the latter seems to be indeed important to determine the optimal screening level policy since it represents the possible gains that the seller may have when selling the good in the outside market. Note that $v_{0}=0$ may represent the case in which the seller has no opportunity to sell the good outside the auction. The most common choice for $v_{0}$ is the appraisal value of 
Figure 3: Private Values Conditional Quantiles

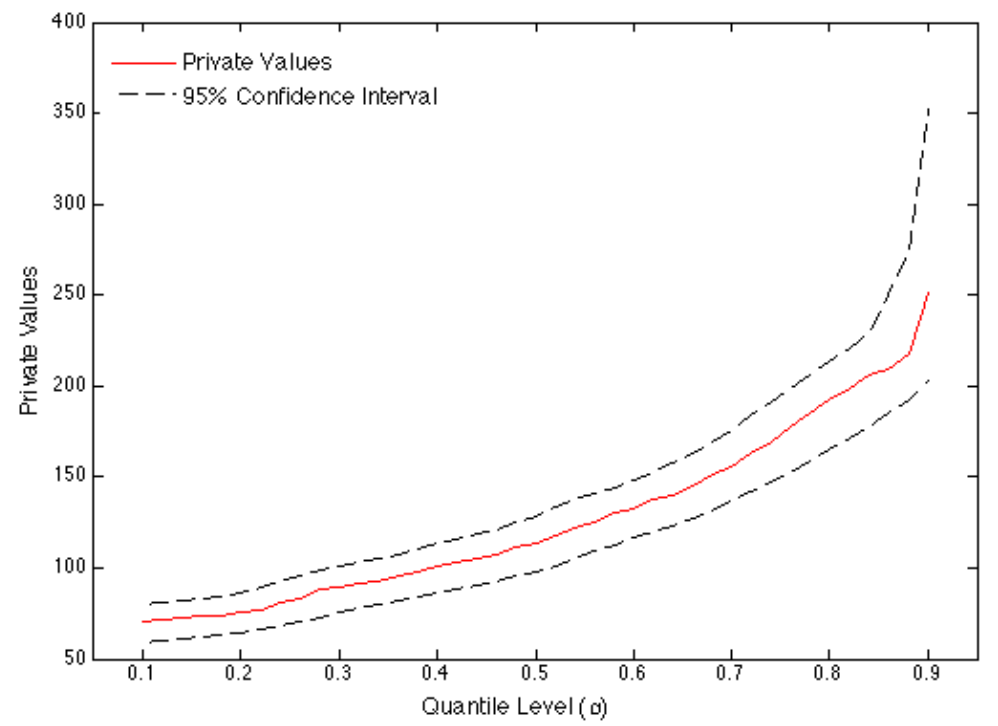

Notes: The estimates are for a median auction. The $95 \%$ confidence intervals were computed by resampling with replacement the $\left(X_{\ell}, b_{w_{\ell}}\right)$-pair in each original subsample $L_{I}$.

Figure 4: Private Values Conditional Quantiles and $X(\tau)$

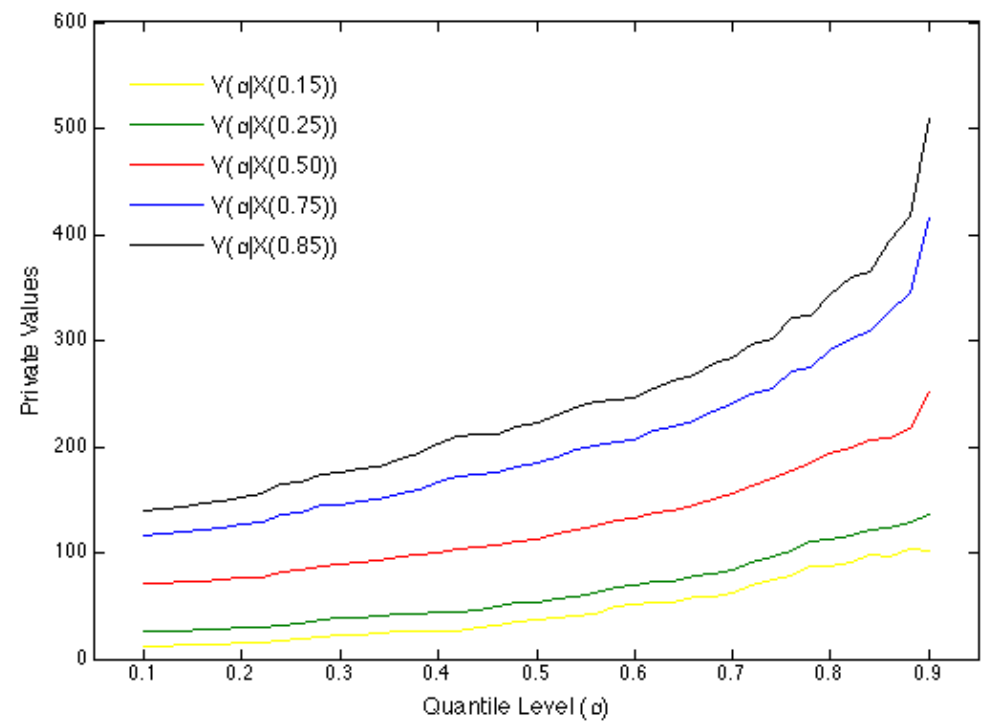

the timber ${ }^{23}$. The results obtained for $v_{0}=$ Appraisal Value (AV) are compared with the

${ }^{23}$ As mentioned in Aradillas-Lopez et al. (2013), the seller's private value may be even lower than the 
Figure 5

Pooled vs Individual $(I=2)$

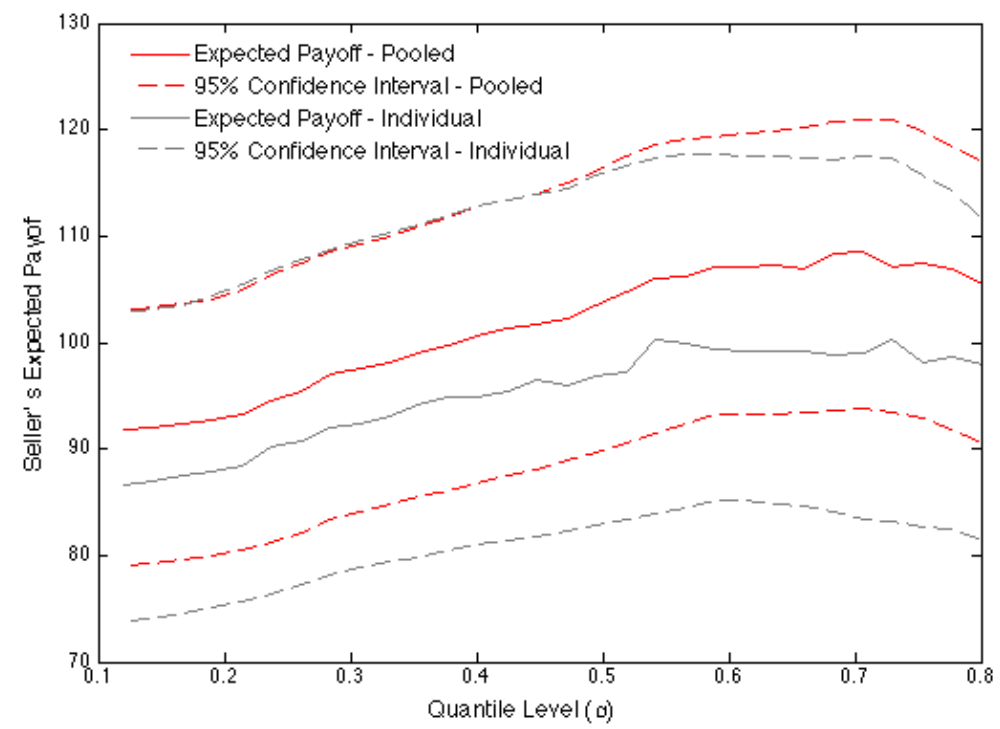

Notes: The estimates are for a median auction. The seller's private value considered here is $v_{0}=A V$, i.e. $v_{0}=\$ 68$ per unit of timber. The $95 \%$ confidence intervals were computed by resampling with replacement the $\left(X_{\ell}, b_{w_{\ell}}\right)$-pair in each original subsample $L_{I}$.

case with no outside option $v_{0}=0$. It is also investigated the effect on the seller's optimal behaviour when the quality and size of the auction change.

Table 8 gives the optimal screening level $\alpha_{r}^{*}$, the corresponding optimal reservation price $r^{*}=V\left(\alpha_{r}^{*} \mid X\right)$ and the seller optimal expected payoff $\Pi\left(\alpha_{r}^{*} \mid X, v_{0}\right)$ for the auctions with quality and size specified by $\tau=0.15,0.50$ and 0.85 and considering both choices of $v_{0}$. Recall that the optimal screening level $\alpha_{r}^{*}$ is chosen as the maximizer of the seller's expected payoff over $\mathcal{A}$. It is expected that $\alpha_{r}^{*}$ that maximize the aggregate expected payoff, as defined in (2.10), is the same as the one maximizing the expected payoff computed for each $I$, i.e. $\alpha_{r}^{*}=\alpha_{r}^{*}(I)$ for all $I$, given the independence result obtained in the test of exclusion appraisal value of the timber if exercising an outside option (through, for example, a lump-sum contract) entails additional cost to the seller. It is also possible that $v_{0}$ is nevertheless higher than the appraisal value since scaled sales require the timber service to measure the timber actually harvested to calculate the payment. Therefore, by exercising the outside option the seller would avoid those costs. 
Figure 6

Pooled vs Individual $(I=3)$

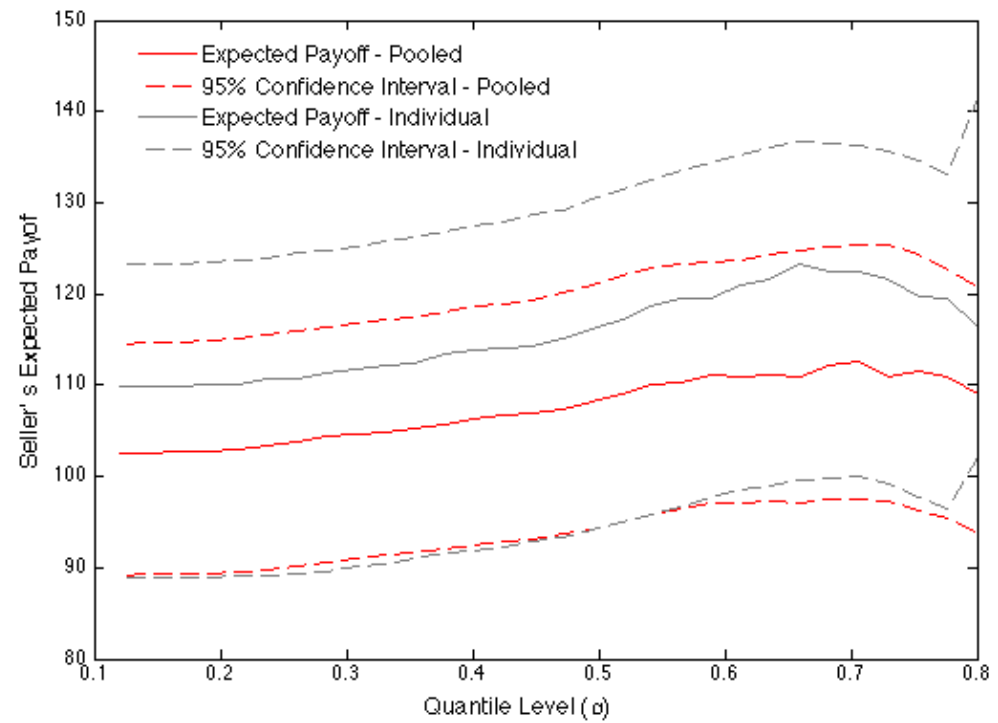

Notes: The estimates are for a median auction. The seller's private value considered here is $v_{0}=A V$, i.e. $v_{0}=\$ 68$ per unit of timber. The $95 \%$ confidence intervals were computed by resampling with replacement the $\left(X_{\ell}, b_{w_{\ell}}\right)$-pair in each original subsample $L_{I}$.

participation restriction. The estimation indeed confirms that $\alpha_{r}^{*}$ is independent upon the number of actual bidders participating in the auction. This will be then reflected in the computation of the corresponding optimal reservation price, which will also not depend upon $I$. Note however, that $\alpha_{r}^{*}$ still depend upon $\left(X, v_{0}\right)$.

A general conclusion from Table 8, which is also expected, is that auctions with higher quality and size provide larger expected payoffs for the seller. The optimal screening level in turn reduces when the quality and size of the auction increase. A possible reason for that is the low heterogeneity among bidders observed in better auctions. Recall from Figure 4 that auctions with low quality and size show a significant increase in the markup over the timber value. Therefore, as seen from Proposition 2.8, the seller has a stronger incentive to use screening for low quality and size auctioned goods. For these low quality and size auctions, the seller private value does not seem to change reservation price recommendations, which, 
Table 8

Optimal Reservation Price

\begin{tabular}{|c|c|c|}
\hline & \multicolumn{2}{|c|}{ Seller's Private Value } \\
\hline & 0 & AV \\
\hline \multirow[t]{2}{*}{$\alpha_{r}^{*}\left(v_{0}, X(0.15)\right)$} & 0.75 & 0.75 \\
\hline & {$[0.52,0.80]^{\mathrm{a}}$} & {$[0.56,0.80]$} \\
\hline \multirow[t]{2}{*}{$V\left(\alpha_{r}^{*} \mid X(0.15)\right)$} & 83.08 & 83.08 \\
\hline & {$[38.93,104.58]$} & {$[45.34,109.03]$} \\
\hline \multirow{2}{*}{$\Pi\left(\alpha_{r}^{*} \mid X(0.15), I=2, v_{0}\right)$} & 32.64 & 39.05 \\
\hline & {$[26.16,44.78]$} & {$[30.26,50.33]$} \\
\hline \multirow[t]{2}{*}{$\Pi\left(\alpha_{r}^{*} \mid X(0.15), I=3, v_{0}\right)$} & 38.98 & 43.80 \\
\hline & {$[30.62,52.92]$} & {$[33.32,56.73]$} \\
\hline \multirow[t]{2}{*}{$\alpha_{r}^{*}\left(v_{0}, X(0.50)\right)$} & 0.28 & 0.71 \\
\hline & {$[0.12,0.36]$} & {$[0.56,0.78]$} \\
\hline \multirow[t]{2}{*}{$V\left(\alpha_{r}^{*} \mid X(0.50)\right)$} & 87.93 & 159.89 \\
\hline & {$[67.73,101.96]$} & {$[123.40,192.43]$} \\
\hline \multirow[t]{2}{*}{$\Pi\left(\alpha_{r}^{*} \mid X(0.50), I=2, v_{0}\right)$} & 91.71 & 108.49 \\
\hline & {$[79.17,102.32]$} & {$[95.11,122.13]$} \\
\hline \multirow[t]{2}{*}{$\Pi\left(\alpha_{r}^{*} \mid X(0.50), I=3, v_{0}\right)$} & 102.93 & 112.50 \\
\hline & {$[99,126.57]$} & {$[89.63,114.26]$} \\
\hline \multirow[t]{2}{*}{$\alpha_{r}^{*}\left(v_{0}, X(0.85)\right)$} & 0.24 & 0.56 \\
\hline & {$[0.12,0.42]$} & {$[0.47,0.80]$} \\
\hline \multirow[t]{2}{*}{$V\left(\alpha_{r}^{*} \mid X(0.85)\right)$} & 163.65 & 243.97 \\
\hline & {$[135.51,213.63]$} & {$[220.03,376.25]$} \\
\hline \multirow[t]{2}{*}{$\Pi\left(\alpha_{r}^{*} \mid X(0.85), I=2, v_{0}\right)$} & 177.66 & 203.26 \\
\hline & {$[163.40,192.73]$} & {$[187.71,223.19]$} \\
\hline \multirow[t]{2}{*}{$\Pi\left(\alpha_{r}^{*} \mid X(0.85), I=3, v_{0}\right)$} & 196.37 & 208.61 \\
\hline & {$[179.89,212.72]$} & {$[191.63,231.26]$} \\
\hline
\end{tabular}

as the expected payoff, remains constant when $v_{0}$ grows from 0 to the appraisal value. This may also be related with the large heterogeneity among the bidders, which resulted in a strong increase in the private value conditional quantiles.

As mentioned in Section 2.2, the probability of trading in the auction with a screening 
level $\alpha_{r}$ is $1-\alpha_{r}^{I}$. Table 9 groups the probabilities of trading in each of the three kinds of auctions for the two choices of $v_{0}$. Note that in the auctions with low quality and size, the probability of selling the good is very low (44\% and $58 \%$ for $I=2$ and $I=3$, respectively). This is because bidders are very heterogeneous and the seller should set a high screening level to avoid low bidders from participating. This somehow carries over for median and higher quality and size auctions when the seller's private value is the appraisal value. Policy recommendations with such low probability of selling may not make sense in practice, especially for goods with a potential high storage cost. Note however that auctions with high quality and size and $I=3$ seem not to be so much affected by this issue.

Table 9: Probability of Trading

\begin{tabular}{cccc}
\hline \hline & & $v_{0}=0$ & $v_{0}=\mathrm{AV}$ \\
\hline$X(0.15)$ & $I=2$ & $44 \%$ & $44 \%$ \\
& $I=3$ & $58 \%$ & $58 \%$ \\
$X(0.50)$ & $I=2$ & $92 \%$ & $50 \%$ \\
& $I=3$ & $98 \%$ & $65 \%$ \\
$X(0.85)$ & $I=2$ & $94 \%$ & $69 \%$ \\
& $I=3$ & $99 \%$ & $82 \%$ \\
\hline
\end{tabular}

By reducing the seller's private value, the probability of trading increase, but such a consideration is mostly theoretical since it is not possible in practice to change the seller's private value. It nevertheless shows that the seller has a high incentive to decrease the optimal screening level when he faces the case in which there is no trade outside the auction. As can be seen, the practical implementation of the auction theory can be sometimes difficult in the sense that usual choices for the seller's private value may lead to recommendation of mechanisms with very low probability of trading. This may question the relevance of considering expected payoff in the maximization process. 


\section{Conclusion}

This paper proposes an identification and estimation approach based on quantile regression to recover the bidders' private values conditional distribution. The quantile regression framework provides a flexible and convenient parametrization of the private value distribution, with an estimation methodology easy to implement and with various specification tests that can be derived. The latter includes tests of dependence between private values and actual number of bidders, linear versus exponential specifications and constancy in the slope coefficients across quantile levels.

The paper shows that a focus on the quantile level of the private values distribution and on the seller's optimal screening level can be both useful for policy recommendations. The former helps newcomer bidders that do not know the market a priori to benchmark their bids in order to achieve a desired probability of winning the auction, whereas the latter provides a better understanding of how appropriate is the recommended policy from a seller's point of view. Both focuses above are new in the empirical auction literature.

The empirical application using timber auctions from the USFS shows that policy recommendations should be carefully examined before practical implementation. The screening level associated with the optimal reservation price is usually high, resulting in a low probability of trading. The analysis of the shape of the private value conditional quantile curves suggests that such inappropriate recommendations are due to a sharp increase in the private value conditional quantiles, which may be evidence of large heterogeneity among the bidders. As a consequence, the seller has a strong incentive to screen bidders' participation by using a high reservation price, leading then to a low probability of selling the auctioned good.

The private values estimated quantile shapes can be genuine but can also be the consequence of a model misspecification. Some other works have also noticed such a high level of the optimal reservation price in timber auctions. Aradillas-Lopez et al. (2013) suggest 
that neglecting private values affiliation can generate high reservation prices. However, their nonparametric methodology may be affected by the curse of dimensionality. In addition, as noted in Roberts and Sweeting (2012), timber auctions include a preliminary selection that can affect the estimated shape of the private value quantile functions. The strong heterogeneity revealed by the estimation of the private value conditional quantile function can also be an indication of asymmetry. As discussed in Cantillon (2008) and Gavious and Minchunk (2012), sellers facing asymmetry have an incentive to increase competition by increasing reservation prices.

However, analysing revenue with a risk neutral seller perspective may not be appropriate to address issues such as high reservation prices and low probability of selling the auctioned

object. The results given in $\mathrm{Hu}$, Matthews and Zou (2010) regarding risk aversion affecting sellers can be useful to provide more relevant reservation price recommendations. Gimenes (2014) proposes a numerical investigation of the variation in the optimal screening level when the seller has a constant relative risk aversion utility function and concludes that considering risk averse sellers is indeed sufficient to achieve reasonable policy recommendations.

\section{Appendix A - Proofs}

\section{A.1 - Proof of Lemma 1:}

From the definition (2.1), the $\alpha$-quantile of the private values conditional distribution must satisfies

$$
F_{v}(V(\alpha \mid X, I) \mid X, I)=\alpha
$$


Under the IPV paradigm, a bidder $i$ with private value $v_{i}$ wins with probability $F_{v}^{I-1}\left(v_{i} \mid X, I\right)$. Therefore, in a quantile setup,

$$
F_{v}^{I-1}(V(\alpha \mid X, I) \mid X, I)=\alpha^{I-1}
$$

which gives the result.

\section{A.2 - Proof of Lemma 2:}

From the definitions of quantiles and $\Psi_{I}(\cdot)$, the $\alpha$-quantile of the winning bids c.d.f. must satisfies $\Psi_{I}\left(F_{v}(B(\alpha \mid X, I) \mid X, I)\right)=\alpha$. Because $\Psi_{I}(\cdot)$ is strictly increasing ${ }^{24}$ in $[0,1]$, the winning bids conditional quantile can be written as

$$
B(\alpha \mid X, I)=F_{v}^{-1}\left(\Psi_{I}^{-1}(\alpha) \mid X, I\right)
$$

where $\Psi_{I}^{-1}(\cdot)$ is the inverse of $\Psi_{I}(\cdot)$. Hence, (2.1) gives $(2.5)$.

\section{A.3 - Proof of Lemma 3:}

If the private values conditional quantile has a quantile regression specification as in (2.4), by Lemma 2 there exists a vector of coefficient $\beta(\alpha \mid I)$, for each $\alpha \in[0,1]$, such that the winning bids conditional quantile satisfies $B(\alpha \mid X, I)=h(X \beta(\alpha \mid I))$. This is a stability property of the quantile regression approach. Since $h(\cdot)$ is continuous and strictly increasing,

${ }^{24}$ The first derivative of $\Psi_{I}(t)$ is always positive for all $t \in(0,1)$ :

$$
\Psi_{I}^{(1)}(t)=\frac{\partial \Psi_{I}(t)}{\partial t}=I(I-1) t^{I-2}(1-t) .
$$


the candidate $\gamma(\cdot \mid I)$ must solve

$$
X \gamma(\alpha \mid I)=X \beta\left(\Psi_{I}(\alpha) \mid I\right)
$$

for all $\mathcal{X}=\{1\} \times \mathcal{Z}$. Since $\mathcal{Z}$ has a nonempty interior by Assumption 4 , this equation has a unique solution and $\gamma(. \mid I)$ is identified for all $I$.

\section{A.4 - Proof of Proposition 4:}

Consider the seller's payoff defined in (2.7). Under assumption 1, the seller possible payoffs are

$$
\pi(r)=\left\{\begin{array}{cl}
v_{0} & \text { if } v_{I: I}<r \\
r \quad & \text { if } v_{I-1: I}<r \leq v_{I: I}, \\
v_{I-1: I} & \text { if } r \leq v_{I-1: I}
\end{array}\right.
$$

where $v_{1: I} \leq \cdots \leq v_{I: I}$ are the ordered private values.

Rewriting $\pi(r)$ using quantiles and the ordered ranks

$$
\alpha_{1: I} \leq \cdots \leq \alpha_{I: I}, \quad v_{i: I}=V\left(\alpha_{i: I}\right) \text { for } i=1, \ldots, I
$$

Recall that the non ordered $\alpha_{i}$ are i.i.d. $\mathcal{U}_{[0,1]}$ random variables. Because $V(\cdot \mid X, I)$ is strictly increasing by assumption 2,

$$
\pi\left(\alpha_{r} \mid X, I\right)= \begin{cases}v_{0}, & \text { if } \alpha_{I: I}<\alpha_{r}, \\ V\left(\alpha_{r} \mid X, I\right), & \text { if } \alpha_{I-1: I}<\alpha_{r} \leq \alpha_{I: I}, \\ V\left(\alpha_{I-1: I} \mid X, I\right), & \text { if } \alpha_{r} \leq \alpha_{I-1: I},\end{cases}
$$

where $\alpha_{r}$ is the level of screening and $V\left(\alpha_{r} \mid X, I\right)$ the reservation price. It follows that the 
seller's expected payoff is

$$
\begin{aligned}
\Pi\left(\alpha_{r} \mid X, I, v_{0}\right)= & v_{0} \mathbb{P}\left(\alpha_{I: I}<\alpha_{r} \mid I\right)+V\left(\alpha_{r} \mid X, I\right) \mathbb{P}\left(\alpha_{I-1: I}<\alpha_{r} \leq \alpha_{I: I} \mid I\right) \\
& +\mathbb{E}\left[V\left(\alpha_{I-1: I} \mid X, I\right) \mathbb{I}\left(\alpha_{r} \leq \alpha_{I-1: I}\right) \mid X, I\right] .
\end{aligned}
$$

Observe that:

(i) $\mathbb{P}\left(\alpha_{I: I}<\alpha_{r} \mid I\right)=\alpha_{r}^{I}$;

(ii) Since $\sum_{i=1}^{I} \mathbb{I}\left(\alpha_{i} \leq \alpha_{r}\right)$ is a binomial distribution with parameters $I$ and $\alpha_{r}$,

$$
\mathbb{P}\left(\alpha_{I-1: I}<\alpha_{r} \leq \alpha_{I: I} \mid I\right)=\mathbb{P}\left(\sum_{i=1}^{I} \mathbb{I}\left(\alpha_{i} \leq \alpha_{r}\right)=I-1 \mid I\right)=I \alpha_{r}^{I-1}\left(1-\alpha_{r}\right)
$$

(iii) Since the c.d.f of $\alpha_{I-1: I}$ given $I$ is $\Psi_{I}(\alpha)$ with p.d.f. $I(I-1) \alpha^{I-2}(1-\alpha), \alpha \in[0,1]$,

$$
\mathbb{E}\left[V\left(\alpha_{I-1: I} \mid X, I\right) \mathbb{I}\left(\alpha_{r} \leq \alpha_{I-1: I}\right) \mid X, I\right]=I(I-1) \int_{\alpha_{r}}^{1} V(\alpha \mid X, I) \alpha^{I-2}(1-\alpha) d \alpha
$$

Therefore, substituting (i)-(iii) into (6.24) gives equation (2.8), which is a quantile version of the seller's expected payoff.

Now, if $\Pi\left(\alpha_{r} \mid X, I, v_{0}\right)$ achieves a maximum for $\alpha_{r}^{*}=\alpha_{r}^{*}\left(X, I, v_{0}\right) \in[0,1], \alpha_{r}^{*}$ must satisfy a first order condition $\frac{\partial}{\partial \alpha}\left\{\Pi\left(\alpha_{r}^{*} \mid X, I, v_{0}\right)\right\}=0$. An expression for the first derivative of $\Pi\left(\alpha_{r}^{*} \mid X, I, v_{0}\right)$ with respect to the rank $\alpha$ is

$$
\begin{aligned}
\frac{\partial}{\partial \alpha}\left\{\Pi\left(\alpha \mid X, I, v_{0}\right)\right\}= & v_{0} I \alpha^{I-1}+V^{(1)}(\alpha \mid X, I) I \alpha^{I-1}(1-\alpha) \\
& +V(\alpha \mid X, I)\left\{I(I-1) \alpha^{I-2}(1-\alpha)-I \alpha^{I-1}\right\} \\
& -V(\alpha \mid X, I) I(I-1) \alpha^{I-2}(1-\alpha) \\
= & I \alpha^{I-1}\left\{v_{0}+V^{(1)}(\alpha \mid X, I)(1-\alpha)-V(\alpha \mid X, I)\right\} .
\end{aligned}
$$


Hence, a candidate for the optimal level of screening $\alpha_{r}^{*}$ is given by

$$
V\left(\alpha_{r}^{*} \mid X, I\right)-V^{(1)}\left(\alpha_{r}^{*} \mid X, I\right)\left(1-\alpha_{r}^{*}\right)=v_{0} .
$$

\section{A.5 - Proof of Theorem 5 and Corollary 6:}

Only the proof of the Theorem is detailed since the proof of the Corollary follows the same steps. Changes needed for the proof of the Corollary are discussed at the end of the section.

I show first that the private values quantile regression estimator in (3.11) is consistent. Since $-1 \leq \partial \rho_{\Psi_{I}(\alpha)}(t) / \partial t \leq 1$, the Taylor inequality gives

$$
\left|\rho_{\Psi_{I}(\alpha)}\left(b_{w_{\ell}}-h\left(X_{\ell} \gamma\right)\right)-\rho_{\Psi_{I}(\alpha)}\left(b_{w_{\ell}}\right)\right| \leq\left|h\left(X_{\ell} \gamma\right)\right| \leq C_{0},
$$

because $X_{\ell} \gamma \in \mathcal{X} \times \Gamma$ compact, and for this reason stays bounded from above. Hence,

$$
\sup _{\gamma \in \Gamma}|\widehat{Q}(\gamma \mid \alpha, I)-Q(\gamma \mid \alpha, I)| \stackrel{\mathbb{P}}{\longrightarrow} 0,
$$

by the Uniform Law of Large Numbers in Newey and McFadden (1994, Lemma 2.4), where $Q(\gamma \mid \alpha, I)$ and $\widehat{Q}(\gamma \mid \alpha, I)$ are defined in section 3. Therefore, by (ii) in Lemma 3 and Newey and McFadden (1994, Theorem 2.1), $\gamma^{*}(\alpha \mid I)$ is uniquely identified and $\widehat{\gamma}(\alpha \mid I) \stackrel{\mathbb{P}}{\longrightarrow} \gamma^{*}(\alpha \mid I)$.

For the sake of brevity, the dependence on $\alpha$ and $I$ is removed, that is $\widehat{Q}(\gamma \mid \alpha, I)$, $Q(\gamma \mid \alpha, I), \widehat{\gamma}(\alpha \mid I)$ and $\gamma^{*}(\alpha \mid I)$ become $\widehat{Q}(\gamma), Q(\gamma), \widehat{\gamma}$ and $\gamma^{*}$. Observe that the first and 
second-order derivatives of $Q(\gamma)$ are

$$
\begin{aligned}
Q_{\gamma}(\gamma)= & \mathbb{E}\left[\left\{\mathbb{I}\left(b_{w}<h(X \gamma)\right)-\Psi_{I}(\alpha)\right\} h^{(1)}(X \gamma) X^{\prime} \mid I\right] \\
= & \mathbb{E}\left[\left\{\mathbb{P}\left(b_{w}<h(X \gamma) \mid X, I\right)-\Psi_{I}(\alpha)\right\} h^{(1)}(X \gamma) X^{\prime} \mid I\right] \\
= & \mathbb{E}\left[\left\{F_{b_{w}}(h(X \gamma) \mid X, I)-\Psi_{I}(\alpha)\right\} h^{(1)}(X \gamma) X^{\prime} \mid I\right], \\
Q_{\gamma \gamma}(\gamma)= & \mathbb{E}\left[f_{b_{w}}(h(X \gamma) \mid X, I) h^{(1)}(X \gamma) X^{\prime} X \mid I\right] \\
& +\mathbb{E}\left[\left\{F_{b_{w}}(h(X \gamma) \mid X, I)-\Psi_{I}(\alpha)\right\} h^{(2)}(X \gamma) X^{\prime} X \mid I\right],
\end{aligned}
$$

as stated in the Theorem. Define

$$
\begin{aligned}
u_{\ell} & =b_{w_{\ell}}-h\left(X_{\ell} \gamma^{*}\right), \\
\widehat{W} & =\frac{1}{L_{I}} \sum_{\ell=1}^{L} \mathbb{I}\left(I_{\ell}=I\right)\left(\mathbb{I}\left(u_{\ell}<0\right)-\Psi_{I}(\alpha)\right) h^{(1)}\left(X_{\ell} \gamma^{*}\right) X_{\ell}^{\prime}, \\
\widehat{R}(\gamma) & =\frac{\sqrt{L_{I}}\left\{\widehat{Q}(\gamma)-\widehat{Q}\left(\gamma^{*}\right)-\left(\gamma-\gamma^{*}\right)^{\prime} \widehat{W}-\left(Q(\gamma)-Q\left(\gamma^{*}\right)\right)\right\}}{\left\|\gamma-\gamma^{*}\right\|},
\end{aligned}
$$

where $\|\cdot\|$ is the usual Euclidean norm. Since $\gamma^{*}$ is an interior point of $\Gamma$ and because $\widehat{\gamma}$ is consistent, the asymptotic normality of the Theorem follows from

$$
\begin{aligned}
& \sqrt{L_{I}} \widehat{W} \stackrel{d}{\rightarrow} \mathcal{N}\left(0, J\left(\gamma^{*} \mid \alpha, I\right)\right), \\
& \sup _{\gamma \in \Gamma ;\left\|\gamma-\gamma^{*}\right\| \leq \epsilon_{L}}\left|\frac{\widehat{R}(\gamma)}{1+\sqrt{L_{I}}\left\|\gamma-\gamma^{*}\right\|}\right|=o_{\mathbb{P}}(1) \text { for any } \epsilon_{L}=o(1),
\end{aligned}
$$

see Theorem 7.1 in Newey and McFadden (1994). I first establish (6.25). Note that the summands in $\widehat{W}$ are centered since

$$
\mathbb{E}\left[\left(\mathbb{I}(u<0)-\Psi_{I}(\alpha)\right) h^{(1)}\left(X \gamma^{*}\right) X^{\prime} \mid I\right]=Q_{\gamma}\left(\gamma^{*}\right)=0,
$$


because $\gamma^{*}$ in the interior of $\Gamma$ minimizes $Q(\cdot)$, and with a variance given $I$ which is

$$
\begin{aligned}
& \mathbb{E}\left[\left(\mathbb{I}(u<0)-\Psi_{I}(\alpha)\right)^{2} h^{(1)}\left(X \gamma^{*}\right)^{2} X^{\prime} X \mid I\right]= \\
& \mathbb{E}\left[\left(\mathbb{P}(u<0 \mid X, I)-2 \Psi_{I}(\alpha) \mathbb{P}(u<0 \mid X, I)+\Psi_{I}(\alpha)^{2}\right) h^{(1)}\left(X \gamma^{*}\right)^{2} X^{\prime} X \mid I\right] \\
& =J\left(\gamma^{*} \mid \alpha, I\right),
\end{aligned}
$$

since $P(u<0 \mid X, I)=F_{b_{w}}\left(h\left(X \gamma^{*}\right) \mid X, I\right)$. Hence (6.25) follows from the Central Limit Theorem.

The rest of the proof consists then in showing (6.26). First some useful notations are introduced. Note that $Q_{\gamma \gamma}(\gamma)=Q_{\gamma \gamma}^{1}(\gamma)+Q_{\gamma \gamma}^{2}(\gamma)$ with

$$
\begin{aligned}
Q_{\gamma \gamma}^{1}(\gamma) & =\mathbb{E}\left[\left\{F_{b_{w}}(h(X \gamma) \mid X, I)-\Psi_{I}(\alpha)\right\} h^{(2)}(X \gamma) X^{\prime} X \mid I\right], \\
Q_{\gamma \gamma}^{2}(\gamma) & =\mathbb{E}\left[f_{b_{w}}(h(X \gamma) \mid X, I) h^{(1)}(X \gamma)^{2} X^{\prime} X \mid I\right] .
\end{aligned}
$$

Write

$$
\begin{aligned}
\widehat{Q}(\gamma)-\widehat{Q}\left(\gamma^{*}\right) & =\frac{1}{L_{I}} \sum_{\ell=1}^{L} \mathbb{I}\left(I_{\ell}=I\right)\left\{\rho_{\Psi_{I}(\alpha)}\left(u_{\ell}-v_{\ell}\right)-\rho_{\Psi_{I}(\alpha)}\left(u_{\ell}\right)\right\} \text { where } \\
v_{\ell} & =v_{\ell}(\delta)=h\left(X_{\ell} \gamma\right)-h\left(X_{\ell} \gamma^{*}\right), \text { where } \delta=\gamma-\gamma^{*}
\end{aligned}
$$

By an identity from Knight (1998),

$$
\begin{aligned}
\rho_{\Psi_{I}(\alpha)}\left(u_{\ell}-v_{\ell}\right)- & \rho_{\Psi_{I}(\alpha)}\left(u_{\ell}\right)= \\
& v_{\ell}\left\{\mathbb{I}\left(u_{\ell}<0\right)-\Psi_{I}(\alpha)\right\}+\int_{0}^{v_{\ell}}\left\{\mathbb{I}\left(u_{\ell} \leq s\right)-\mathbb{I}\left(u_{\ell} \leq 0\right)\right\} d s .
\end{aligned}
$$


Hence, for $\delta=\gamma-\gamma^{*}$,

$$
\widehat{Q}(\gamma)-\widehat{Q}\left(\gamma^{*}\right)-\left(\gamma-\gamma^{*}\right)^{\prime} \widehat{W}-\left(Q(\gamma)-Q\left(\gamma^{*}\right)\right)=\sum_{j=1}^{5} \bar{\Delta}_{j}(\delta),
$$

where $\bar{\Delta}_{j}(\delta)=\sum_{\ell=1}^{L} \Delta_{j \ell}(\delta) / L_{I}$ with

$$
\begin{aligned}
& \Delta_{1 \ell}(\delta)=\mathbb{I}\left(I_{\ell}=I\right)\left[\left(v_{\ell}-h^{(1)}\left(X_{\ell} \gamma^{*}\right) X_{\ell} \delta\right)\left\{\mathbb{I}\left(u_{\ell}<0\right)-\Psi_{I}(\alpha)\right\}\right.\left.-\mathbb{E}\left[\left(v_{\ell}-h^{(1)}\left(X_{\ell} \gamma^{*}\right) X_{\ell} \delta\right)\left\{\mathbb{I}\left(u_{\ell}<0\right)-\Psi_{I}(\alpha)\right\} \mid I_{\ell}\right]\right], \\
& \Delta_{2 \ell}(\delta)=\mathbb{I}\left(I_{\ell}=I\right)\left[\mathbb{E}\left[\left(v_{\ell}-h^{(1)}\left(X_{\ell} \gamma^{*}\right) X_{\ell} \delta\right)\left\{\mathbb{I}\left(u_{\ell}<0\right)-\Psi_{I}(\alpha)\right\} \mid I_{\ell}\right]\right.\left.-\frac{\delta^{\prime} Q_{\gamma \gamma}^{1}\left(\gamma^{*}\right) \delta}{2}\right], \\
& \Delta_{3 \ell}(\delta)=\mathbb{I}\left(I_{\ell}=I\right)\left[\mathbb{E}\left[\int_{0}^{v_{\ell}}\left\{\mathbb{I}\left(u_{\ell} \leq s\right)-\mathbb{I}\left(u_{\ell} \leq 0\right)\right\} d s \mid I_{\ell}\right]-\frac{\delta^{\prime} Q_{\gamma \gamma}^{2}\left(\gamma^{*}\right) \delta}{2}\right], \\
& \Delta_{4 \ell}(\delta)=\mathbb{I}\left(I_{\ell}=I\right)\left[\int_{0}^{v_{\ell}}\left\{\mathbb{I}\left(u_{\ell} \leq s\right)-\mathbb{I}\left(u_{\ell} \leq 0\right)\right\} d s\right. \\
&\left.-\mathbb{E}\left[\int_{0}^{v_{\ell}}\left\{\mathbb{I}\left(u_{\ell} \leq s\right)-\mathbb{I}\left(u_{\ell} \leq 0\right)\right\} \mid I_{\ell}\right]\right], \\
& \Delta_{5 \ell}(\delta)=\mathbb{I}\left(I_{\ell}=I\right)\left[Q(\gamma)-Q\left(\gamma^{*}\right)-\frac{\delta^{\prime} Q_{\gamma \gamma}^{1}\left(\gamma^{*}\right) \delta}{2}-\frac{\delta^{\prime} Q_{\gamma \gamma}^{2}\left(\gamma^{*}\right) \delta}{2}\right] .
\end{aligned}
$$

Now, since $v_{\ell}=h\left(X_{\ell}\left(\gamma^{*}+\delta\right)\right)-h\left(X_{\ell} \gamma\right)$ satisfies,

$$
\begin{aligned}
v_{\ell} & =X_{\ell} \delta \int_{0}^{1} h^{(1)}\left(X_{\ell}\left(\gamma^{*}+t \delta\right)\right)(1-t) d t, \\
v_{\ell}-h^{(1)}\left(X_{\ell} \gamma^{*}\right) X_{\ell} \delta & =\frac{\delta^{\prime} X_{\ell}^{\prime} X_{\ell} \delta}{2} \int_{0}^{1} h^{(2)}\left(X_{\ell}\left(\gamma^{*}+t \delta\right)\right)(1-t) d t,
\end{aligned}
$$


then

$$
\begin{aligned}
\frac{\partial}{\partial \delta}\left[\frac{v_{\ell}-h^{(1)}\left(X_{\ell} \gamma^{*}\right) X_{\ell} \delta}{\|\delta\|}\right]= & \frac{v_{\ell}}{\|\delta\|}-\frac{\delta\left(v_{\ell}-h^{(1)}\left(X_{\ell} \gamma^{*}\right) X_{\ell} \delta\right)}{\|\delta\|^{2}} \\
= & \frac{\delta}{\|\delta\|} X_{\ell} \int_{0}^{1} h^{(1)}\left(X_{\ell}\left(\gamma^{*}+t \delta\right)\right)(1-t) d t- \\
& \delta \frac{\delta^{\prime} X_{\ell}^{\prime} X_{\ell} \delta}{2\|\delta\|^{2}} \int_{0}^{1} h^{(2)}\left(X_{\ell}\left(\gamma^{*}+t \delta\right)\right)(1-t) d t
\end{aligned}
$$

This first differential is bounded when $\|\delta\| \leq 1$. Hence the set of functions $\left\{\Delta_{1 \ell}(\delta) /\|\delta\|,\|\delta\| \leq 1\right\}$ can be covered with a number less than $O\left(\epsilon^{-C}\right)$ of functional brackets with length $\epsilon$. To see that the same holds for $\left\{\Delta_{4 \ell}(\delta) /\|\delta\|,\|\delta\| \leq 1\right\}$, observe

$$
\begin{gathered}
\frac{1}{\|\delta\|} \int_{0}^{v_{\ell}}\left\{\mathbb{I}\left(u_{\ell} \leq s\right)-\mathbb{I}\left(u_{\ell} \leq 0\right)\right\} d s=\frac{v_{\ell}}{\|\delta\|} \int_{0}^{1}\left\{\mathbb{I}\left(u_{\ell} \leq s+t v_{\ell}\right)-\mathbb{I}\left(u_{\ell} \leq 0\right)\right\} d t \\
=\left(X_{\ell} \frac{\delta}{\|\delta\|}\right) \times\left(\int_{0}^{1} h^{(1)}\left(X_{\ell}\left(\gamma^{*}+t \delta\right)\right)(1-t) d t\right) \\
\times\left(\int_{0}^{1}\left\{\mathbb{I}\left(u_{\ell} \leq s+t v_{\ell}\right)-\mathbb{I}\left(u_{\ell} \leq 0\right)\right\} d t\right) .
\end{gathered}
$$

Each of the three functions of $\delta$ in this product can be covered with $O\left(\epsilon^{-C}\right)$ of functional brackets with length $\epsilon$, implying that the same covering property holds for $\left\{\Delta_{4 \ell}(\delta) /\|\delta\|,\|\delta\| \leq 1\right\}$. Hence, since $\left\{\Delta_{j \ell}(\delta) /\|\delta\|,\|\delta\| \leq \epsilon_{L}\right\}$ admits an envelope $\Delta_{j}\left(X_{\ell}\right)$ satisfying $E\left[\boldsymbol{\Delta}_{j}^{2}\left(X_{\ell}\right)\right]=$ $o(1), j=1,4$, Lemma 19.38 in van der Vaart (1998) gives that, with $\Gamma_{\delta}=\left\{\delta ; \gamma^{*}+\delta \in \Gamma\right\}$

$$
\mathbb{E}\left[\max _{\delta \in \Gamma_{\delta} ;\|\delta\| \leq \epsilon_{L}}\left|\sqrt{L_{I}} \frac{\bar{\Delta}_{j}(\delta)}{\|\delta\|}\right| I_{\ell}, 1 \leq \ell \leq L\right]=o_{\mathbb{P}}(1), \quad j=1,4
$$

This gives, by the Markov inequality,

$$
\max _{\delta \in \Gamma_{\delta} ;\|\delta\| \leq \epsilon_{L}}\left|\sqrt{L_{I}} \frac{\bar{\Delta}_{j}(\delta)}{\|\delta\|}\right|=o_{\mathbb{P}}(1), \quad j=1,4
$$


Now elementary expansions give, for the items in $\Delta_{j \ell}(\delta), j=2,3,5$, uniformly in $\ell$ since $X_{\ell}$ lies in a compact set,

$$
\begin{aligned}
& \mathbb{I}\left(I_{\ell}=I\right) \mathbb{E}\left[\left(v_{\ell}-h^{(1)}\left(X_{\ell} \gamma^{*}\right) X_{\ell} \delta\right)\left\{\mathbb{I}\left(u_{\ell}<0\right)-\Psi_{I}(\alpha)\right\} \mid I_{\ell}\right]= \\
& \mathbb{I}\left(I_{\ell}=I\right) \mathbb{E}\left[\left(\frac{h^{(2)}\left(X_{\ell} \gamma^{*}\right) \delta^{\prime} X_{\ell} X_{\ell} \delta}{2}+o\left(\|\delta\|^{2}\right)\right)\left\{\mathbb{P}\left(u_{\ell}<0 \mid X_{\ell}, I_{\ell}\right)-\Psi_{I}(\alpha)\right\} \mid I_{\ell}\right] \\
& =\mathbb{I}\left(I_{\ell}=I\right) \frac{\delta^{\prime} Q_{\gamma \gamma}^{1}\left(\gamma^{*}\right) \delta}{2}+o\left(\|\delta\|^{2}\right), \\
& \mathbb{I}\left(I_{\ell}=I\right) \mathbb{E}\left[\int_{0}^{v_{\ell}}\left\{\mathbb{I}\left(u_{\ell} \leq s\right)-\mathbb{I}\left(u_{\ell} \leq 0\right)\right\} d s \mid I_{\ell}\right]= \\
& \mathbb{I}\left(I_{\ell}=I\right) \mathbb{E}\left[\int_{0}^{v_{\ell}}\left\{\mathbb{P}\left(u_{\ell} \leq s \mid X_{\ell}, I_{\ell}\right)-\mathbb{P}\left(u_{\ell} \leq 0 \mid X_{\ell}, I_{\ell}\right)\right\} d s \mid I_{\ell}\right]= \\
& \mathbb{I}\left(I_{\ell}=I\right) \mathbb{E}\left[\int_{0}^{v_{\ell}}\left\{f_{b_{w}}\left(h\left(X_{\ell} \gamma^{*}\right) \mid X_{\ell}, I_{\ell}\right)+o(1)\right\} s d s \mid I_{\ell}\right]= \\
& \mathbb{I}\left(I_{\ell}=I\right) \mathbb{E}\left[\left\{f_{b_{w}}\left(h\left(X_{\ell} \gamma^{*}\right) \mid X_{\ell}, I_{\ell}\right)+o(1)\right\} \frac{v_{\ell}^{2}}{2} \mid I_{\ell}\right]= \\
& \mathbb{I}\left(I_{\ell}=I\right) \mathbb{E}\left[f_{b_{w}}\left(h\left(X_{\ell} \gamma^{*}\right) \mid X_{\ell}, I_{\ell}\right) \frac{\left(h^{(1)}\left(X_{\ell} \gamma^{*}\right) X_{\ell}\right)^{2}}{2} \mid I_{\ell}\right]+o\left(\|\delta\|^{2}\right) \\
& \quad=\mathbb{I}\left(I_{\ell}=I\right) \frac{\delta^{\prime} Q_{\gamma \gamma}^{2}\left(\gamma^{*}\right) \delta}{2}+o\left(\|\delta\|^{2}\right), \\
& Q(\gamma)-Q\left(\gamma^{*}\right)-\frac{\delta^{\prime} Q_{\gamma \gamma}^{1}\left(\gamma^{*}\right) \delta}{2}-\frac{\delta^{\prime} Q_{\gamma \gamma}^{2}\left(\gamma^{*}\right) \delta}{2}=o\left(\|\delta\|^{2}\right) .
\end{aligned}
$$

This gives by definition of $\bar{\Delta}_{j}(\delta), j=2,3,5$,

$$
\max _{\delta \in \Gamma_{\delta} ;\|\delta\| \leq \epsilon_{L}}\left|\frac{\bar{\Delta}_{j}(\delta)}{\|\delta\|^{2}}\right|=o_{\mathbb{P}}(1), \quad j=2,3,5 .
$$


Therefore (6.27), (6.28), $\gamma-\gamma^{*}=\delta$, and $\widehat{R}(\gamma)=\sqrt{L_{I}} \sum_{j=1}^{5} \bar{\Delta}_{j}(\delta)$ imply

$$
\begin{aligned}
\sup _{\gamma \in \Gamma ;\left\|\gamma-\gamma^{*}\right\| \leq \epsilon_{L}}\left|\frac{\widehat{R}(\gamma)}{1+\sqrt{L_{I}}\left\|\gamma-\gamma^{*}\right\|}\right| \leq & 2 \max _{j=1,4} \max _{\delta \in \Gamma_{\delta} ;\|\delta\| \leq \epsilon_{L}}\left|\sqrt{L_{I}} \frac{\bar{\Delta}_{j}(\delta)}{\|\delta\|}\right| \\
& +3 \max _{j=1,3,5} \max _{\delta \in \Gamma_{\delta} ;\|\delta\| \leq \epsilon_{L}}\left|\frac{\bar{\Delta}_{j}(\delta)}{\|\delta\|^{2}}\right| \\
= & O_{\mathbb{P}}(1) .
\end{aligned}
$$

Hence (6.26) is true.

For Corrollary 6, the first derivative of $\widehat{Q}^{0}(\gamma)=\widehat{Q}(\gamma \mid \alpha)$ is

$$
\widehat{Q}_{\gamma}^{0}(\gamma)=\frac{1}{L} \sum_{\ell=1}^{L}\left(\mathbb{I}\left(b_{w_{\ell}} \leq h\left(X_{\ell} \gamma\right)\right)-\Psi_{I_{\ell}}(\alpha)\right) h^{(1)}\left(X_{\ell} \gamma\right) X_{\ell}^{\prime}
$$

Let $\gamma^{*}=\gamma(\alpha)$. As seen from the proof of Theorem 5, the matrix $J\left(\gamma^{*} \mid \alpha\right)$ is the variance of $\widehat{W}^{0}=L^{1 / 2} \widehat{Q}_{\gamma}^{0}\left(\gamma^{*}\right)$ and because $\mathbb{E}\left[\widehat{W}^{0}\right]=0$ asymptotic normality follows. Hence $J\left(\gamma^{*} \mid \alpha\right)$ is equal to

$$
\begin{gathered}
\mathbb{E}\left[\left(\mathbb{I}\left(b_{w} \leq h\left(X \gamma^{*}\right)\right)-2 \mathbb{I}\left(b_{w} \leq h\left(X \gamma^{*}\right)\right) \Psi_{I}(\alpha)+\Psi_{I}^{2}(\alpha)\right) h^{(1)}\left(X \gamma^{*}\right) X^{\prime} X\right]= \\
\mathbb{E}\left[\left(\mathbb{P}\left(b_{w} \leq h\left(X \gamma^{*}\right) \mid X, I\right)-2 \mathbb{P}\left(b_{w} \leq h\left(X \gamma^{*}\right) \mid X, I\right) \Psi_{I}(\alpha)+\Psi_{I}^{2}(\alpha)\right)\right. \\
\left.\times h^{(1)}\left(X \gamma^{*}\right) X^{\prime} X\right]
\end{gathered}
$$

which gives the expression of the Corollary since $\mathbb{P}\left(b_{w} \leq h\left(X \gamma^{*}\right) \mid X, I\right)=F_{b_{w}}\left(h\left(X \gamma^{*}\right) \mid X, I\right)$. Now, similar computations give that

$$
\begin{aligned}
Q_{\gamma \gamma}^{0}\left(\gamma^{*}\right)= & \mathbb{E}\left[\widehat{Q}_{\gamma \gamma}^{0}\left(\gamma^{*}\right)\right] \\
= & \mathbb{E}\left[\left(f_{b_{w}}\left(h\left(X \gamma^{*}\right) \mid X, I\right)-\Psi_{I_{\ell}}(\alpha)\right) h^{(1)}\left(X \gamma^{*}\right) X^{\prime} X\right] \\
& +\mathbb{E}\left[\left(F_{b_{w}}\left(h\left(X \gamma^{*}\right) \mid X, I\right)-\Psi_{I_{\ell}}(\alpha)\right) h^{(2)}\left(X \gamma^{*}\right) X^{\prime} X\right]
\end{aligned}
$$




\section{Appendix B - Random Weighting Bootstrap Method}

This section describes the random weighting bootstrap method used in the tests of exclusion participation restriction and constancy of the slope coefficients. Let $\widehat{Q}_{H_{0}}\left(\widehat{\gamma}_{H_{0}} \mid \alpha, I\right)$ and $\widehat{Q}_{H_{1}}\left(\widehat{\gamma}_{H_{1}} \mid \alpha, I\right)$ be the optimized value of the quantile regression objective function under the null and alternative hypothesis, respectively. The M-test statistic defined in Rao and Zhao (1992), Wang and Zhou (2004) and Zhao, Wu and Yang (2007) is

$$
M=L_{I}\left[\widehat{Q}_{H_{0}}\left(\widehat{\gamma}_{H_{0}} \mid \alpha, I\right)-\widehat{Q}_{H_{1}}\left(\widehat{\gamma}_{H_{1}} \mid \alpha, I\right)\right]
$$

Let $\pi_{\ell}$ be i.i.d. random weights multinomially distributed with parameters $\left(L_{I}, 1 / L_{I}\right)$. Define the random weighting empirical objective function under the null and alternative hypotheses as $\widehat{Q}_{H_{0}}\left(\gamma_{H_{0}} \mid \alpha, I, \pi\right)$ and $\widehat{Q}_{H_{1}}\left(\gamma_{H_{1}} \mid \alpha, I, \pi\right)$, where

$$
\widehat{Q}_{H_{j}}\left(\gamma_{H_{j}} \mid \alpha, I, \pi\right)=\frac{1}{L_{I}} \sum_{\ell=1}^{L_{I}} \mathbb{I}\left(I_{\ell}=I\right) \pi_{\ell} \rho_{\Psi_{I}(\alpha)}\left(b_{w_{\ell}}-h\left(X_{\ell} \gamma\right)\right),
$$

for $j=\{0,1\}$. The aforementioned reference suggests to random weighting bootstrap the M-statistic (6.29) by using the random weights $\pi_{\ell}$. Let $b$ be the index for a draw $\pi^{b}$ of the weights. For each $j=\{0,1\}$, define

$$
\widehat{\gamma}_{H_{j}}^{b}(\alpha \mid I)=\arg \min _{\gamma_{H_{j}} \in \Gamma} \widehat{Q}_{H_{j}}\left(\gamma_{H_{j}} \mid \alpha, I, \pi^{b}\right)
$$


Then, the bth draw of the bootstrapped statistic is

$$
\begin{aligned}
M^{b}=L_{I}\left[\widehat{Q}_{H_{0}}\left(\widehat{\gamma}_{H_{0}}^{b} \mid \alpha, I, \pi^{b}\right)\right. & \left.-\widehat{Q}_{H_{1}}\left(\widehat{\gamma}_{H_{1}}^{b} \mid \alpha, I, \pi^{b}\right)\right] \\
& -L_{I}\left[\widehat{Q}_{H_{0}}\left(\widehat{\gamma}_{H_{0}} \mid \alpha, I, \pi^{b}\right)-\widehat{Q}_{H_{1}}\left(\widehat{\gamma}_{H_{1}} \mid \alpha, I, \pi^{b}\right)\right]
\end{aligned}
$$

where $\widehat{\gamma}_{H_{j}}$ are the estimators obtained from the initial population.

The null hypothesis $H_{0}$ is rejected at a significance level $\tau$ if $M$ is larger than the sample $(1-\tau)$-quantile of the bootstrapped statistic $M^{1}, \cdots, M^{B}$ and accepted otherwise, where $B$ is the number of bootstrap replications.

\section{Appendix C - Monte-Carlo Experiments}

This section presents some Monte Carlo experiments used to illustrate the hypotheses tests and their performance in terms of size. For all the experiments below, let $\mathcal{A}=\{0.50,0.60,0.70,0.80\}$.

\section{C.1 - Exclusion Participation Restriction.}

Data Generating Process. Two samples of auction covariates and error terms are generated. One sample corresponds to $L_{2}$ auctions with $I=2$ actual bidders and the other to $L_{3}$ auctions with $I=3$, where $L_{2}=L_{3}=100$. For the auction covariates, $L_{I}$ random Normal $(0,1)$ variables are generated and replicated $I$ times. The $I \times L_{I}$ error terms are randomly generated considering three different parametric distributions: Uniform $(0,1)$, Standard Exponential and Standard Normal. The bidders' private values are generated via two possible regression specifications: linear $v_{i \ell}=1+X_{1 \ell}+X_{2 \ell}+u_{i \ell}$ and exponential $v_{i \ell}=\exp \left(0.3+0.3 X_{1 \ell}+0.3 X_{2 \ell}+u_{i \ell}\right)^{25}$. The winning bid is given by the second-highest

\footnotetext{
${ }^{25}$ The values of the intercept and slope coefficients are not an important issue and were chosen simply to speed up the estimation process.
} 
private value: $b_{w_{\ell}}=v_{I-1: I, \ell}$.

Estimation and Test. The winning bids are used to estimate $\gamma(\alpha \mid I=2)$ and $\gamma(\alpha \mid I=3)$, via the individual quantile regression estimator (3.11), and $\gamma(\alpha)$, via the pooled quantile regression estimator (3.17), for all $\alpha \in \mathcal{A}$. The statistic

$$
M=\widehat{Q}(\widehat{\gamma} \mid \alpha)-\sum_{I \in\{2,3\}} \widehat{Q}(\widehat{\gamma}(\alpha \mid I) \mid \alpha, I) L_{I} / L
$$

is computed for each quantile level $\alpha$ and summed over $\mathcal{A}$.

Bootstrap. $L_{2}$ and $L_{3}$ i.i.d. random weights $\pi$ multinomially distributed with parameters $\left(L_{I}, 1 / L_{I}\right)$ are generated and used to estimate $\widehat{Q}\left(\widehat{\gamma}^{b}(\alpha) \mid \alpha, \pi^{b}\right)$ and $\widehat{Q}\left(\widehat{\gamma}^{b}(\alpha \mid I) \mid \alpha, I, \pi^{b}\right)$ for each bootstrap draw $b$, where the former corresponds to the pooled optimized objective function and the latter to the individual one. I then compute the random weighting bootstrap statistic

$$
\begin{aligned}
M^{b}=\left[\widehat{Q}\left(\widehat{\gamma}^{b}(\alpha) \mid \alpha, \pi^{b}\right)-\right. & \left.\sum_{I \in\{2,3\}} \widehat{Q}\left(\widehat{\gamma}^{b}(\alpha \mid I) \mid \alpha, I, \pi^{b}\right) L_{I} / L\right]- \\
& {\left[\widehat{Q}\left(\widehat{\gamma}(\alpha) \mid \alpha, \pi^{b}\right)-\sum_{I \in\{2,3\}} \widehat{Q}\left(\widehat{\gamma}(\alpha \mid I) \mid \alpha, I, \pi^{b}\right) L_{I} / L\right], }
\end{aligned}
$$

where $\widehat{\gamma}(\alpha)$ and $\widehat{\gamma}(\alpha \mid I)$ are the estimates of the original population, and sum over the quantile levels. The number of bootstrap replications is $B=500$.

Bootstrap Critical Values. The significance level $\tau$ is chosen for the test. The bootstrap critical value $c^{*}(\tau)$ is an approximation of the $c(\tau)$ critical value of the test statistic $M$ and is given by the $(1-\tau)$ th quantile of the empirical distribution of the bootstrapped statistic.

Rejection Rule. If $M<c^{*}(\tau)$, then the null hypothesis is not rejected at $\tau \%$ significance level. 
Table 10

Rejection Probability - Linear Specification

\begin{tabular}{lrrr}
\hline \hline Nominal Level & Std. Normal & Uniform $(0,1)$ & Std. Exponential \\
\hline $1 \%$ & $1.06 \%$ & $1.18 \%$ & $0.87 \%$ \\
$5 \%$ & $5.09 \%$ & $5.47 \%$ & $3.62 \%$ \\
$10 \%$ & $9.84 \%$ & $10.84 \%$ & $7.30 \%$ \\
\hline \multicolumn{2}{c}{ The number of bootstrap replications is $B=500 ;$ The experiment was repeated } \\
$N=10,000$ times; The set of prescribed quantiles is $\mathcal{A}=\{0.50,0.60,0.70,0.80\}$.
\end{tabular}

Table 11

Rejection Probability - Exponential Specification

\begin{tabular}{lrrr}
\hline \hline Nominal Level & Std. Normal & Uniform $(0,1)$ & Std. Exponential \\
\hline $1 \%$ & $1.32 \%$ & $1.62 \%$ & $1.17 \%$ \\
$5 \%$ & $5.14 \%$ & $6.16 \%$ & $4.61 \%$ \\
$10 \%$ & $9.94 \%$ & $11.54 \%$ & $9.34 \%$ \\
\hline \multicolumn{2}{c}{ The number of bootstrap replications is $B=500 ;$ The experiment was repeated } \\
$N=10,000$ times; The set of prescribed quantiles is $\mathcal{A}=\{0.50,0.60,0.70,0.80\}$.
\end{tabular}

Rejection Probability. The procedure above is repeated $N=10,000$ times and the proportion of rejections of the null hypothesis is computed.

The results from the Monte Carlo experiment above are given in Tables 10 and 11. The rejection probabilities are very close to the nominal levels in both cases. It is slightly undersized in the case in which the errors follow a standard exponential distribution and the model has a linear specification.

\section{C.2 - Choice of Specification}

Data Generating Process. As in the previous experiment, two samples of auction covariates and error term with $L_{2}$ and $L_{3}$ auctions are generated, where $L_{2}=L_{3}=200$. The auction covariates are random Standard Normal variables that are replicated $I$ times. For the error terms, the following distributions are considered: Standard Normal, Uniform $(0,1)$ and Standard Exponential. The bidders' private values are generated via two possible regression specifications: linear $v_{i \ell}^{\mathrm{L}}=\gamma_{0}+\gamma_{1} Z_{1 \ell}+u_{i \ell}$ and exponential $v_{i \ell}^{\mathrm{E}}=\exp \left(\gamma_{0}+\gamma_{1} Z_{1 \ell}+u_{i \ell}\right)$, 
where $\left(\gamma_{0}, \gamma_{1}\right)=(1,1)$ in the case the errors are normally distributed and $\left(\gamma_{0}, \gamma_{1}\right)=(0,0.5)$ when the errors are either uniformly or exponentially distributed ${ }^{26}$. Under the null hypothesis, the exponential specification is equivalent to the linear specification for a given $\alpha_{0} \in \mathcal{A}$ and dominates the latter for all $\alpha \in \mathcal{A} \backslash\left\{\alpha_{0}\right\}$. In order to have equivalence at $\alpha_{0} \in \mathcal{A}$, a private value $v_{i \ell}$, which is a linear combination of both private values above, is created: $v_{i \ell}=\lambda v_{i \ell}^{\mathrm{L}}+(1-\lambda) v_{i \ell}^{\mathrm{E}}$. To find the weight $\lambda$ that satisfies the equivalence property, I split $\lambda \in[0,1]$ into 1,000 equal spaces and compute the relative difference between both optimized objective functions,

$$
\Delta\left(\alpha, \lambda_{t}\right)=\frac{\left(\widehat{Q}_{\mathrm{E}}\left(\widehat{\gamma}_{\mathrm{E}} \mid \alpha, \lambda_{t}\right)-\widehat{Q}_{\mathrm{L}}\left(\widehat{\gamma}_{\mathrm{L}} \mid \alpha, \lambda_{t}\right)\right)}{\widehat{Q}_{\mathrm{L}}\left(\widehat{\gamma}_{\mathrm{L}} \mid \alpha, \lambda_{t}\right)}
$$

for $t=\{1, \cdots, 1,000\} . \lambda^{*}$ is chosen over $t$ such that to minimize $\Delta\left(\alpha, \lambda_{t}\right)$ for a given $\alpha$. The equivalence property must hold for a given $\alpha_{0} \in \mathcal{A}$ and the exponential specification should be better than the linear one for all $\alpha \in \mathcal{A} \backslash\left\{\alpha_{0}\right\}$. Therefore, $\lambda_{\max }^{*}$ is chosen such that $\sup _{\alpha \in \mathcal{A}} \Delta\left(\alpha, \lambda^{*}\right)=0$. The winning bid is given by the second-highest private value: $b_{w_{\ell}}=v_{I-1: I, \ell}$

Estimation and Test. A DGP using $\lambda_{\max }^{*}$ is generated and the test statistic $\widehat{\text { Vuong }}$ computed as in (4.22).

Bootstrap. The pair $\left(X_{\ell}, b_{w_{\ell}}\right)$ is resampled with replacement from its joint sample distribution in each original subsample $L_{I}$. The number of bootstrap replications is $B=500$.

Bootstrapping the Test Statistic. For each bootstrap replication $b$, the recentered bootstrapped test statistic $\widehat{\operatorname{Vuong}}^{b}$ is computed as

$$
\widehat{\operatorname{Vuong}}^{b}=\sup _{\alpha \in \mathcal{A}} \sqrt{L}\left\{\left(\widehat{Q}_{\mathrm{E}}\left(\widehat{\gamma}_{\mathrm{E}}^{b} \mid \alpha\right)-\widehat{Q}_{\mathrm{L}}\left(\widehat{\gamma}_{\mathrm{L}}^{b} \mid \alpha\right)\right)-\left(\widehat{Q}_{\mathrm{E}}\left(\widehat{\gamma}_{\mathrm{E}} \mid \alpha\right)-\widehat{Q}_{\mathrm{L}}\left(\widehat{\gamma}_{\mathrm{L}} \mid \alpha\right)\right)\right\}
$$

\footnotetext{
${ }^{26}$ The values of the intercept and slope parameters were chosen according to the distribution of the errors in an attempt to find a DGP that satisfies the equivalence property for a given quantile level.
} 
Table 12

Rejection Probability - Test of Specification

\begin{tabular}{lrrr}
\hline \hline Nominal Level & Std. Normal & Uniform $(0,1)$ & Std. Exponential \\
\hline $1 \%$ & $2.23 \%$ & $0.94 \%$ & $0.91 \%$ \\
$5 \%$ & $5.67 \%$ & $4.50 \%$ & $4.22 \%$ \\
$10 \%$ & $8.31 \%$ & $9.04 \%$ & $8.22 \%$ \\
\hline$\lambda_{\text {max }}^{*}$ & 0.6547 & 0.5846 & 0.5095 \\
\hline \multicolumn{2}{l}{ The number of bootstrap replications is $B=500 ;$ The experiment was repeated } \\
$N=10,000$ times; The set of prescribed quantiles is $\mathcal{A}=\{0.50,0.60,0.70,0.80\} ;$ \\
It is assumed independence of the private values distribution upon $I$ in this Monte \\
Carlo experiment.
\end{tabular}

where $\widehat{\gamma}_{\mathrm{E}}$ and $\widehat{\gamma}_{\mathrm{L}}$ are the estimates of the original population generated by $\lambda_{\max }^{*}$.

The bootstrap critical values, rejection rule and rejection probability are computed as in Appendix C.1. Table 12 gives the rejection probabilities and the values for $\lambda_{\max }^{*}$ used in each experiment. The test performs well although a bit undersized. That is because the supremum is used over the quantiles in the formula, which means that the rejection probability could be higher for a given quantile level.

\section{C.3 - Constancy of the Slope Coefficients}

Data Generating Process. As in the previous two experiments, two samples of auction covariates and error terms with $L_{2}$ and $L_{3}$ auctions are generated, where $L_{2}=L_{3}=100$. For the auction covariates, $L_{I}$ random Standard Normal variables are generated and replicated $I$ times. The $I \times L_{I}$ error terms are randomly generated considering the parametric distributions: Uniform $(0,1)$, Standard Exponential and Standard Normal. The private values are generated using a linear regression model due to the results of the empirical application, i.e. $v_{i \ell}=0+Z_{\ell}+u_{i \ell}$. The winning bid is given by the second-highest private value: $b_{w_{\ell}}=v_{I-1: I, \ell}$.

Estimation and Test. The winning bids are used to estimate $\left(\gamma_{0_{k}}, \gamma_{1}\right), k=1, \cdots, K$, via the CQR estimator defined in (3.20), and $\gamma(\alpha)$ via the pooled quantile regression estimator (3.17). Under the null hypothesis, the CQR population objective function equals the 
Table 13

Rejection Probability - Constancy of the Slope Coefficients

\begin{tabular}{lrrr}
\hline \hline Nominal Level & Std. Normal & Uniform $(0,1)$ & Std. Exponential \\
\hline $1 \%$ & $1.25 \%$ & $1.15 \%$ & $1.09 \%$ \\
$5 \%$ & $6.25 \%$ & $6.73 \%$ & $5.59 \%$ \\
$10 \%$ & $11.74 \%$ & $12.27 \%$ & $10.95 \%$ \\
\hline
\end{tabular}

The number of bootstrap replications is $B=500$; The experiment was repeated $N=10,000$ times; The set of prescribed quantiles is $\mathcal{A}=\{0.50,0.60,0.70,0.80\}$; It is assumed independence of the private values distribution upon $I$ in this Monte Carlo experiment.

average of the individual objective functions over $\mathcal{A}$. The M-statistic

$$
M=L\left[\widehat{Q}_{\mathrm{CQR}}\left(\widehat{\gamma}_{\mathrm{CQR}}\right)-\frac{1}{K} \sum_{k=1}^{K} \widehat{Q}\left(\widehat{\gamma} \mid \alpha_{k}\right)\right]
$$

is computed, where $K=4$ since $\mathcal{A}=\{0.50,0.60,0.70,0.80\}$.

Bootstrap. $L_{2}$ and $L_{3}$ i.i.d. random weights $\pi$ multinomially distributed with parameters $\left(L_{I}, 1 / L_{I}\right)$ are generated and used to estimate $\widehat{Q}_{\mathrm{CQR}}\left(\widehat{\gamma}_{\mathrm{CQR}}^{b} \mid \pi^{b}\right)$ and $\widehat{Q}\left(\widehat{\gamma}^{b}\left(\alpha_{k}\right) \mid \alpha_{k}, \pi^{b}\right)$ at each bootstrap draw $b$ and quantile level $\alpha_{k}$. The random weight bootstrap statistic is then computed as

$$
\begin{aligned}
M^{b}=L\left[\widehat{Q}_{\mathrm{CQR}}\left(\widehat{\gamma}_{\mathrm{CQR}}^{b} \mid \pi^{b}\right)\right. & \left.-\frac{1}{K} \sum_{k=1}^{K} \widehat{Q}\left(\widehat{\gamma}^{b}\left(\alpha_{k}\right) \mid \alpha_{k}, \pi^{b}\right)\right]- \\
L & {\left[\widehat{Q}_{\mathrm{CQR}}\left(\widehat{\gamma}_{\mathrm{CQR}} \mid \pi^{b}\right)-\frac{1}{K} \sum_{k=1}^{K} \widehat{Q}\left(\widehat{\gamma}\left(\alpha_{k}\right) \mid \alpha_{k}, \pi^{b}\right)\right], }
\end{aligned}
$$

where $\widehat{\gamma}_{\mathrm{CQR}}$ and $\widehat{\gamma}\left(\alpha_{k}\right)$ are the estimates of the original population. The number of bootstrap replications is $B=500$.

The bootstrap critical values, rejection rule and rejection probability are computed as in Appendix C.1. Table 13 gives the rejection probabilities of the test. The test performs well although a bit oversized, which is due to the tolerance level asigned for the convergence of the algorithm. 


\section{References}

[1] Aradillas-Lopez, A., A. Gandhi \& D. Quint (2013). Identification and inference in ascending auctions with correlated private values. Econometrica, 81, 489-534.

[2] Athey, S., J. Levin (2001). Information and competition in U.S. forest service timber auctions. Journal of Political Economy, 109, 375-417.

[3] Athey, S., J. Levin \& E. Seira (2011). Comparing open and sealed bid auctions: evidence from timber auctions. The Quarterly Journal of Economics, 126, 207-257.

[4] Athey, S. \& P. Haile (2002). Identification of standard auction models. Econometrica, 70, 2107-2140.

[5] Baldwin, L., R. Marshall \& J.F. Richard (1997). Bidder collusion at Forest Service Timber Sales. Journal of Political Economy, 105, 657-699.

[6] Bikhchandani, S., P.A. Haile \& J.G. Riley (2002). Symmetric separating equilibria in English auctions. Games and Economic Behavior, 38, 19-27.

[7] Buchinsky, M. (1995). Estimating the asymptotic covariance matrix for quantile regression models: a Monte Carlo study. Journal of Econometrics, 68, 303-338.

[8] Campo, S., E. Guerre, I. Perrigne \& Q. Vuong (2011). Semiparametric estimation of first-price auctions with risk averse bidders. Review of Economic Studies, 78, $112-147$.

[9] Canay, I.A.(2011). A simple approach to quantile regression for panel data. The Econometrics Journal, 14, 368-386.

[10] Cantillon, E.(2008). The effect of bidders' asymmetries on expected revenue in auctions. Games and Economic Behavior, 62, 1-25.

[11] Chernozhukov, V. \& C. Hansen(2006). Instrumental quantile regression inference for structural and treatment effect models. Journal of Econometrics, 132, 491-525.

[12] Donald, S. \& H. PaArsch (1993). Piecewise pseudo-maximum likelihood estimation in empirical models of auctions. International Economic Review, 34, 121-148.

[13] Donald, S. \& H. PAarsch (1996). Identification, estimation, and testing in parametric empirical models of auctions within the independent private values paradigm. Econometric Theory, 12, 517-567.

[14] Enache, A. \& J.P Florens (2012). Identification and estimation in a third-price auction model. Working Paper, Paris School of Economics. 
[15] Galvao JR., A. (2011). Quantile regression for dynamic panel data with fixed effects. Journal of Econometrics, 164, 142-157.

[16] Gavious, A. \& Y. Minchuk (2012). A note on the effect of asymmetry on revenue in second-price auctions. International Game Theory Review, 14, Issue 03.

[17] Gentry, M. \& T. Li (2012). Identification in auctions with selective entry. Working Paper. Revised and resubmitted to Econometrica.

[18] Gimenes, N. (2014). To Sell or not to Sell? An Empirical Analysis of the Optimal Reservation Price in Ascending Timber Auctions. Working Paper, Department of Economics, University of Sao Paulo.

[19] Gimenes, N. \& E. Guerre (2014). Parametric and nonparametric quantile regression methods for first-price auction: a signal approach. Working Paper, Queen Mary, University of London.

[20] Guerre, E., I. Perrigne \& Q. Vuong (2000). Optimal nonparametric estimation of first-price auctions. Econometrica, 68, 525-574.

[21] Guerre, E. \& C. Sabbah (2012). Uniform bias study and Bahadur representation for local polynomial estimators of the conditional quantile function. Econometric Theory, 28, 87-129.

[22] Haile, P.A. (2001). Auctions with resale markets: an application to U.S. Forest Service Timber Sales. American Economic Review, 91, 399-427.

[23] Haile, P.A. \& E. Tamer (2003). Inference with an incomplete model of English auctions. Journal of Political Economy, 111, 1-51.

[24] Haile, P.A., H. Hong \& M. Shum (2003). Nonparametric tests for common values in first-price sealed-bid auctions. Cowles Foundation discussion paper.

[25] Hastie, T.J. \& R.J. Tibshirani (1990). Generalized additive models. London: Chapman \& Hall/CRC. Monographs on Statistics \& Applied Probability.

[26] Horowitz, J. \& V. Spokoiny (2001). An adaptive, rate-optimal test of a parametric mean-regression model against a nonparametric alternative. Econometrica, 69, 599-631.

[27] Hu, A., S.A. Matthews \& L. Zou (2010). Risk aversion and optimal reserve prices in first- and second-price auctions. Journal of Economic Theory, 145, 1188-1202.

[28] Knight, K.(1998). Limiting distributions for $L_{1}$ regression estimators under general conditions. Annals of Statistics, 26, 755-770. 
[29] Koenker, R. (2005). Quantile Regression. Cambridge Books, Cambridge University Press.

[30] Koenker, R. \& G. Bassett (1978). Regression quantiles. Econometrica, 46, 33-50.

[31] Koenker, R. \& J. Machado (1999). Goodness of fit and related inference processes for quantile regression. Journal of the American Statistical Association, 94, 1296-1310.

[32] Kullback, S. \& R.A. Leibler (1951). On information and sufficiency. Annals of Mathematical Statistics, 22, 79-86.

[33] Krishna, V. (2010). Auction Theory. Academic Press.

[34] Laffont, J.J., H. Ossard \& Q. VuOng (1995). Econometrics of first-price auctions. Econometrica 63, 953-980.

[35] Levin, D. \& J. Smith (1994). Equilibrium in auctions with entry. The American Economic Review, 84, 585-599.

[36] Li, T. \& I. Perrigne (2003). Timber sale auctions with random reserve prices. Review of Economics and Statistics, 85, 189-200.

[37] Li, T., I. Perrigne \& Q. Vuong (2003). Semiparametric estimation of the optimal reserve price in first-price auctions. Journal of Business and Economic Statistics, 21, 53-64.

[38] Li, T. \& X. Zheng (2009). Entry and competition effects in first-price auctions: theory and evidence from procurement auctions. Review of Economic Studies, 76, 1397-1429.

[39] Li, T. \& X. Zheng (2012). Information acquisition and/or bid preparation: a structural analysis of entry and bidding in timber sale auctions. Journal of Econometrics, 168, 29-46.

[40] Lu, J. \& I. Perrigne (2008). Estimating risk aversion from ascending and sealedbid auctions: the case of timber auction data. Journal of Applied Econometrics, 23, 871-896.

[41] Marmer, V., \& A. Shneyerov (2012). Quantile-based nonparametric inference for first-price auctions. Journal of Econometrics, 167, 345-357.

[42] Marmer, V., A. Shneyerov \& P. Xu (2013). What model for entry in first-price auctions? A nonparametric approach. Journal of Econometrics, 176, 46-58.

[43] Myerson, R.(1981). Optimal auction design. Mathematics of Operations Research, 6, 58-63.

[44] Newey, W. \& D. McFAdDen (1994). Large sample estimation and hypothesis testing. In Handbook of Econometrics, 4, Ch. 36. Elsevier Science. 
[45] PaArsch, H. (1992). Deciding between the common and private value paradigms in empirical models of auctions. Journal of Econometrics, 51, 191-215.

[46] Powell, J.L. (1991). Estimation of monotonic regression models under quantile restrictions, in Nonparametric and Semiparametric Methods in Econometrics, (ed. by W. Barnett, J.L. Powell and G. Tauchen), Cambridge: Cambridge University Press.

[47] RaO, C.R., \& L.C. ZhaO (1992). Approximation to the distribution of M-estimates in linear models by randomly weighted bootstrap. Sankhya 54, 323-331.

[48] Rezende, L. (2008). Econometrics of auctions by least squares. Journal of Applied Econometrics 23, 925-948.

[49] Riley, J.G., \& W.F. Samuelson (1981). Optimal auctions. The American Economic Review 71, 381-392.

[50] Roberts, J. \& A. Sweeting (2012). When Should Sellers Use Auctions? Working Paper, Duke University.

[51] Silverman, B.W. (1998). Density estimation for statistics and data analysis. London: Chapman \& Hall/CRC. Monographs on Statistics \& Applied Probability.

[52] VAn Der VAart, A.W. (1998). Asymptotic Statistics. Cambridge University Press, Cambridge.

[53] VuOnG, Q. (1989). Likelihood ratio test for model selection and non-nested hypotheses. Econometrica, 57, 307-333.

[54] Wang, X.M. \& W. Zhou (2004). Bootstrap approximation to the distribution of M-estimates in a linear model. Acta Mathematica Sinica, 20, 93-104.

[55] Whang, Y.J.(2006). Consistent specification testing for quantile regression mod- els, in Econometric Theory and Practice: Frontiers of Analysis and Applied Research, ed. by D. Corbae, S. Durlauf, and B. Hansen, Cambridge University Press.

[56] White, H. (1982). Maximum Likelihood Estimation of Misspecified Models. Econometrica, 50, 1-25.

[57] Zhao, L., X. Wu \& Y. YAng (2007). Approximation by random weighting method for M-test in linear models. Science in China Series A: Mathematics, 50, 87-99.

[58] Zheng, J.(1998). A consistent nonparametric test of parametric regression models under conditional quantile restrictions. Econometric Theory, 14, 123-138.

[59] Zou, H. \& M. YuAN (2008). Composite quantile regression and the oracle model selection theory. The Annals of Statistics, 36, 1108-1126. 